\title{
Einstein Gravity Explorer-a medium-class fundamental physics mission
}

\author{
S. Schiller • G. M. Tino • P. Gill • C. Salomon - U. Sterr • E. Peik • \\ A. Nevsky • A. Görlitz • D. Svehla • G. Ferrari • N. Poli • \\ L. Lusanna • H. Klein • H. Margolis • P. Lemonde • P. Laurent • \\ G. Santarelli - A. Clairon · W. Ertmer · E. Rasel • J. Müller · L. Iorio · \\ C. Lämmerzahl · H. Dittus • E. Gill • M. Rothacher • F. Flechner • \\ U. Schreiber • V. Flambaum • Wei-Tou Ni • Liang Liu • Xuzong Chen • \\ Jingbiao Chen · Kelin Gao $\cdot$ L. Cacciapuoti • R. Holzwarth • \\ M. P. Heß • W. Schäfer
}

Received: 3 December 2007 / Accepted: 6 October 2008 / Published online: 29 November 2008 (C) Springer Science + Business Media B.V. 2008

\footnotetext{
S. Schiller $(\varangle) \cdot$ A. Nevsky · A. Görlitz

Institut für Experimentalphysik,

Heinrich-Heine-Universität, 40225 Düsseldorf, Germany

e-mail: step.schiller@uni-duesseldorf.de

G. M. Tino · G. Ferrari · N. Poli

Dipartimento di Fisica and LENS Laboratory,

Università di Firenze, CNR/INFM, INFN - Sez. di Firenze,

50019 Sesto Fiorentino, Italy

P. Gill · H. Klein · H. Margolis

National Physical Laboratory, Teddington TW11 0LW, UK

C. Salomon

Laboratoire Kastler Brossel, Ecole Normale Supérieure,

24 rue Lhomond 75231 Paris, France

U. Sterr · E. Peik

Physikalisch-Technische Bundesanstalt, Bundesallee 100,

38116 Braunschweig, Germany
}

D. Svehla

Institute of Astronomical and Physical Geodesy, Technische Universität München,

Arcisstrasse 21, 80333 Munich, Germany

L. Lusanna

INFN, Sez. di Firenze, Polo Scientifico, 50019 Sesto Fiorentino, Italy

P. Lemonde · P. Laurent · G. Santarelli · A. Clairon

LNE-SYRTE, Observatoire de Paris, 61 Avenue de l'observatoire, 75014 Paris, France

W. Ertmer · E. Rasel

Institut für Quantenoptik, Leibniz Universitaet Hannover,

Welfengarten 1, 30167 Hannover, Germany 
Abstract The Einstein Gravity Explorer mission (EGE) is devoted to a precise measurement of the properties of space-time using atomic clocks. It tests one of the most fundamental predictions of Einstein's Theory of General Relativity, the gravitational redshift, and thereby searches for hints of quantum effects in gravity, exploring one of the most important and challenging frontiers in fundamental physics. The primary mission goal is the measurement of the gravitational redshift with an accuracy up to a factor $10^{4}$ higher than the best current result. The mission is based on a satellite carrying cold atombased clocks. The payload includes a cesium microwave clock (PHARAO), an optical clock, a femtosecond frequency comb, as well as precise microwave time transfer systems between space and ground. The tick rates of the clocks are continuously compared with each other, and nearly continuously with clocks on earth, during the course of the 3-year mission. The highly elliptic orbit of the satellite is optimized for the scientific goals, providing a large variation in the gravitational potential between perigee and apogee. Besides the fundamental physics results, as secondary goals EGE will establish a global

J. Müller

Institut Für Erdmessung, Leibniz Universität Hannover,

Schneiderberg 50, 30167 Hannover, Germany

L. Iorio

Sezione di Pisa, INFN, Viale Unità di Italia 68, 70125 Bari, Italy

C. Lämmerzahl · H. Dittus

Centre of Applied Space technology and Microgravity (ZARM),

University of Bremen, Am Fallturm, 28359 Bremen, Germany

E. Gill

Department of Earth Observation and Space Systems,

Delft University of Technology, Kluyverweg 1,

2629 HS Delft, The Netherlands

M. Rothacher · F. Flechner

GeoForschungsZentrum, Potsdam, Germany

U. Schreiber

Fundamentalstation Wettzell, 93444 Bad Koetzting, Germany

V. Flambaum

University of New South Wales, Sydney, Australia

W.-T. Ni

Purple Mountain Observatory, Chinese Academy of Sciences,

2, Beijing W. Road, Nanjing, 210008, China

L. Liu

Shanghai Institute of Optics and Fine Mechanics,

Chinese Academy of Sciences Jiading, Shanghai 201800, China 
reference frame for the Earth's gravitational potential and will allow a new approach to mapping Earth's gravity field with very high spatial resolution. The mission was proposed as a class-M mission to ESA's Cosmic Vision Program 2015-2025.

Keywords Gravitational redshift - Gravitational frequency shift • Atomic clock - Optical clock $\cdot$ Equivalence principle $\cdot$ Local position invariance $\cdot$ Special relativity - Lorentz invariance $\cdot$ General relativity $\cdot$ Geoid • Frequency transfer $\cdot$ Microwave link $\cdot$ Gravitational potential

\section{Abbreviations}

AOM acoustooptic modulator/shifter

ACES atomic clock ensemble in space

CNES centre national etudes spatiales

CCR corner cube reflector

EEP Einstein Equivalence Principle

EGE Einstein Gravity Explorer

EO electro-optic modulator

ESA European space agency

FCDP frequency comparison and distribution unit

GGOS global geodetic observing system

GNSS global navigation satellite system

GPS global positioning system

G/S ground station(s)

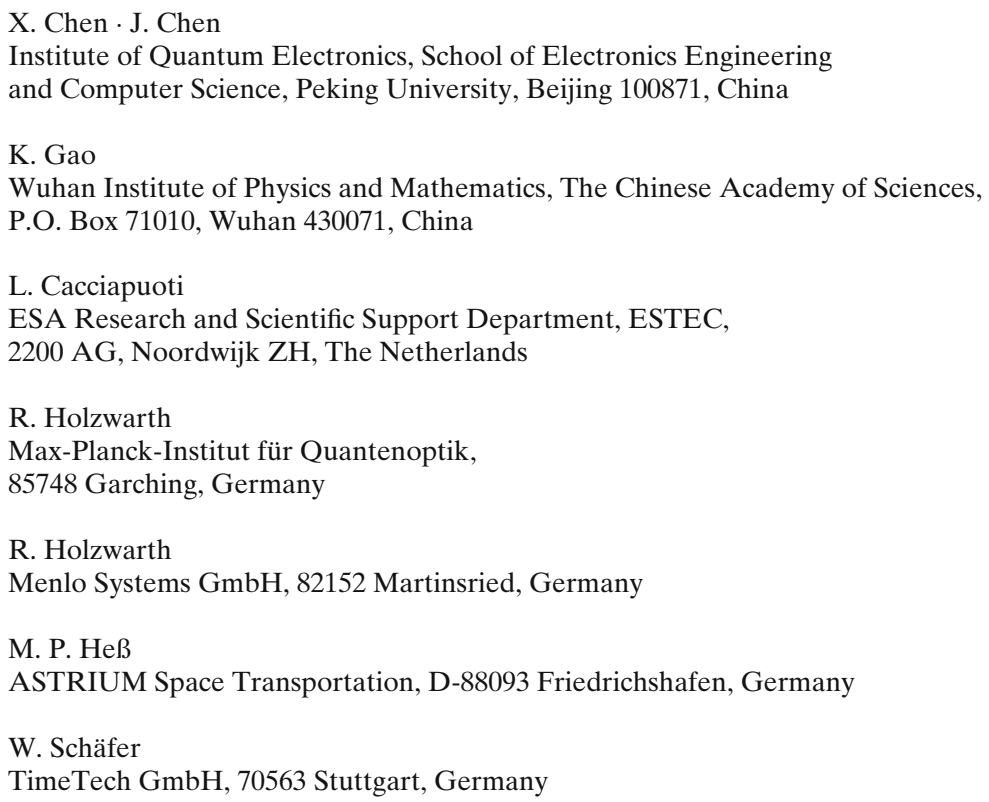




$\begin{array}{ll}\text { GR } & \text { General Relativity } \\ \text { GRACE } & \begin{array}{l}\text { gravity recovery and climate experiment } \\ \text { international space station }\end{array} \\ \text { ISS } & \text { Local Position Invariance } \\ \text { LPI } & \text { microwave link } \\ \text { MWL } & \text { microwave-optical local oscillator } \\ \text { MOLO } & \text { monitoring and control } \\ \text { M\&C } & \text { optical frequency comb } \\ \text { OFC } & \text { Projet d'horloge atomique par refroidissement d'atomes en } \\ \text { PHARAO } & \text { orbite } \\ \text { PLL } & \text { phase-locked loop } \\ \text { PTB } & \text { Physikalisch-Technische Bundesanstalt } \\ \text { PRARE } & \text { Precise Range And Range-Rate Equipment } \\ \text { QCD } & \text { quantum chromodynamics } \\ \text { RF } & \text { radiofrequency } \\ \text { Rx } & \text { receiver } \\ \text { SLR } & \text { satellite laser ranging } \\ \text { SME } & \text { standard model extension } \\ \text { TC } & \text { telecommand } \\ \text { TM } & \text { telemetry } \\ \text { T\&F } & \text { time and frequency } \\ \text { ULE } & \text { ultralow-expansion glass } \\ \text { UHV } & \text { ultra-high vacuum } \\ \text { UTC } & \text { universal time coordinated } \\ \text { USO } & \text { ultrastable quartz oscillator } \\ \text { XPLC } & \text { onboard computer/control system } \\ & \end{array}$

\section{Introduction}

Einstein's theory of General Relativity is a cornerstone of our current description of the physical world. It is used to describe the motion of bodies ranging in size from satellites to galaxy clusters, the propagation of electromagnetic waves in the presence of massive bodies, and the dynamics of the Universe as a whole. In general, the measurement of general relativistic effects is very challenging, due to their small size [1,2]. Thus, only few of its predictions have been tested with high accuracy, for example the time delay of electromagnetic waves via the Cassini mission [3], and the existence of gravitational waves by observation of binary star systems. The accuracy of the determination of these effects is at the $20 \mathrm{ppm}$ level.

The situation is similar for one of the most fascinating effects predicted by General Relativity and other metric theories, the slowing of clocks in a gravitational field (gravitational time dilation, gravitational redshift). The gravitational redshift was measured with $7 \times 10^{-5}$ relative inaccuracy in the 1976 Gravity Probe-A experiment [4] by comparing a ground clock with a similar clock on a rocket as the height changed. The most performing clocks 
available at the time, hydrogen masers, were used for this experiment. The ACES (Atomic Clock Ensemble in Space) mission planned to fly on the ISS in 2014 seeks to improve the determination by a factor 25 , by using a cold atom clock (PHARAO) and a hydrogen maser [5, 6]. Recent progress on cold atom clocks in the optical domain and in optical technology enable performing this fundamental test with orders of magnitude better sensitivity.

Although it has been very successful so far, General Relativity, as well as numerous other alternative or more general theories proposed in the course of the development of theoretical physics, are classical theories. As such, they are fundamentally incomplete, because they do not include quantum effects. In fact, it has not yet been possible to develop a theory of gravity that includes quantum mechanics in a satisfactory way, although enormous effort has been devoted to this goal. Such a theory would represent a crucial step towards a unified theory of all fundamental forces of nature. Several approaches have been proposed and are currently under investigation (e.g. string theory). A full understanding of gravity will require observations or experiments that determine the relationship of gravity with the quantum world. This topic is a prominent field of activity and includes the current studies of dark energy.

The Einstein Gravity Explorer is a fundamental physics mission dedicated to this question; its primary task is to measure the gravitational frequency shift with unprecedented accuracy. The EGE mission uses a satellite on a highly elliptic orbit (see Fig. 1). The ratio of the frequencies of two on-board clocks and the ratios between satellite and ground clocks are the main observables.

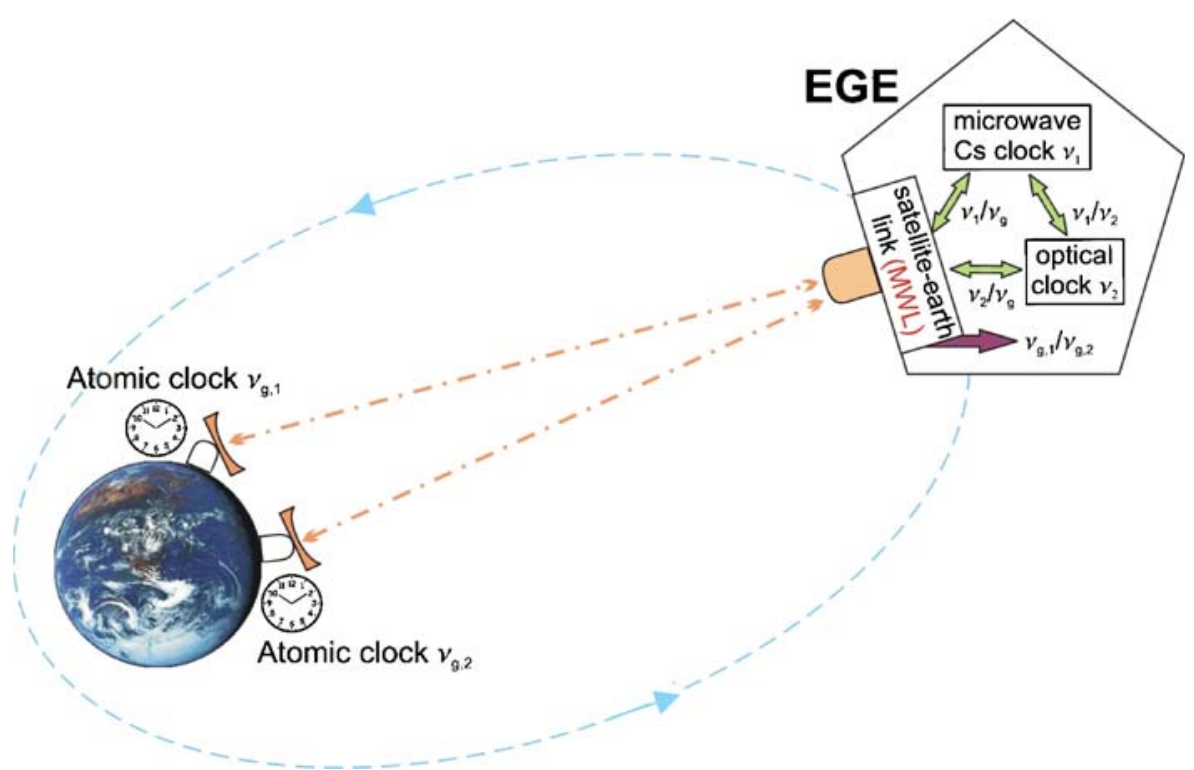

Fig. 1 General concept of the EGE mission. Clocks on the satellite and on ground are intercompared as the satellite orbits the Earth on a highly elliptic orbit 
These can be interpreted toward tests of General Relativity (GR) and of competing metric theories of gravity and as tests for the existence of new fields associated to matter (see Section 3). The outcome of the mission will be either a confirmation of GR and metric theories within the accuracy provided by the instruments, or the discovery of a deviation. In the latter case, the mission would provide a first indication of the breakdown of current (classical) gravitational physics theories and could pave the way towards a unified theory of all forces.

\section{Scientific objectives}

2.1 Summary of science objectives

2.1.1 Fundamental physics science objectives $\left(1 \mathrm{ppm}=1\right.$ part in $10^{6}$, $1 \mathrm{ppb}=1$ part in $10^{9}$ )

Primary objectives

High-precision measurement of the earth gravitational frequency shift at $25 \mathrm{ppb}$ accuracy, a factor 3,000 improvement

First precise measurement of the sun gravitational frequency shift at $1 \mathrm{ppm}$ level, a factor $2 \times 10^{4}$ improvement

Test of space and time variability of fundamental constants with accuracy $30 \mathrm{ppb}$

First search for neutron scalar charge, with accuracy $30 \mathrm{ppb}$

Test of anomalous coupling of matter to the Standard Model quantum fields

Secondary objectives

Tests of Lorentz Invariance in the matter and photon sector (factor 20 improvement)

Contribution towards establishing a new definition of the unit of time

\subsubsection{Spin-off to other fields (outside fundamental physics)}

Establishment of a new approach to the determination of the geopotential, with geoid height resolution of a few $\mathrm{cm}$ Comparison of distant terrestrial clocks at the $10^{-18}$ level

Demonstration of clock and link technology for future applications, e.g. in precision spacecraft navigation

Demonstration on high performance real-time range determination with precision 25 times (based on code phase) to 1,000 times (based on carrier 
phase) higher compared to systems onboard previous missions (ERS-2, TOPEX POSEIDON)

Comparison of three different orbit determination systems: LaserRanging, $\mu \mathrm{m}$-precision microwave ranging, GPS-based orbit determination

Atmospheric signal propagation study, precise determination of third order ionospheric term

Monitoring stability of the Galileo/GPS/Glonass time scales

\subsection{Gravitational frequency shift measurement and search for new physics}

The primary science goal of EGE is a measurement and test of the gravitational frequency shift in the weak-field limit, as predicted by metric theories of gravitation. These theories also predict that the shift is independent of the type of clock used to measure it. The comparison of the frequencies $v$ of two identical clocks 1,2 , operating at different locations $x_{1}, x_{2}$, yields a frequency ratio

$$
v_{2}\left(x^{\prime}\right) / v_{1}\left(x^{\prime}\right) \cong 1+\left(U\left(x_{2}\right)-U\left(x_{1}\right)\right) / c^{2} .
$$

Here, $v_{i}\left(x^{\prime}\right)$ is the frequency of clock $i$ located at $x_{i}$, as observed (measured) at a particular location $x^{\prime}$ where the comparisons are performed. The comparison location can be arbitrary but will often be $x_{1}$, or $x_{2}$, or both. $U$ is the gravitational potential, $U(x)=-G M /|x|$, in case of a spherically symmetric body of mass $M$. In short, time runs (or clocks tick) more slowly the closer the system/clock is to a massive body. Stationary clocks and observers and weak gravitational fields have been assumed in Eq. 1.

Through its measurement, EGE searches for a possible violation of the gravitational frequency shift. A violation may be described phenomenologically by a dependence of one or more fundamental constants on the gravitational potential, $X=X\left(U / c^{2}\right)$, where $X$ is a generic dimensionless fundamental constant or a dimensionless combination of fundamental constants. Such a dependence is a violation of the principle of Local Position Invariance (LPI), which states that the outcome of local, nongravitational experiments is independent of where and when in the Universe they are performed. If a constant depends on the gravitational potential, then the results of those laboratorytype (i.e. local) experiments that depend on that constant, will also depend on the location, namely on the location of the laboratory with respect to the mass distribution generating the potential, and will violate LPI.

LPI is one of the three cornerstones of the Einstein Equivalence Principle (EEP), the others being the Weak Equivalence Principle and Local Lorentz Invariance. It is a common belief that the EEP may not be absolutely valid therefore spatial dependence and time dependence of fundamental constants have become a very intense research topic in the last decade, with experiments undertaken worldwide to search for violations of various aspects of EEP. For 
example, extensive studies of atomic and molecular spectra of gas clouds in the distant Universe are currently undertaken to search for a difference of these parameters compared to today's values (see e.g. [7-11] and references therein). Also, some differences in Big Bang nucleosynthesis data and calculations can be naturally explained by a variation of fundamental constants $[12,13]$.

The EGE mission is thus a probe of possible space-dependence of fundamental constants. The search may help to point the way toward a unified theory of all forces, reveal hypothetical extra dimensions in our Universe or the existence of many sub-Universes with different physics, chemistry and biology. Indeed, theories unifying all forces, applied to cosmology, suggest space and time dependence of the coupling constants [14-16]. Another argument for spatial variation of fundamental constants comes from the anthropic principle. There must be a fine tuning of fundamental constants which allows humans and any life to appear. Life in its present form is not consistent with fundamental constants whose values only slightly differ from the actual ones. This fine tuning can be naturally explained by the spatial variation of the fundamental constants: we appeared in the area of the Universe where the values of the fundamental constants are consistent with our existence.

In the Standard Model there are three main fundamental dimensionless parameters determining the structure and energy levels of stable matter (atoms, molecules): the electromagnetic fine structure constant $\alpha$ and the two ratios of electron mass $m_{\mathrm{e}}$ and light quark mass $m_{\mathrm{q}}$ to the strong QCD energy scale $\Lambda_{\mathrm{QCD}}, X_{\mathrm{e}, \mathrm{q}}=m_{\mathrm{e}, \mathrm{q}} / \Lambda_{\mathrm{QCD}}$. The fundamental masses $m_{\mathrm{e}}$ and $m_{\mathrm{q}}$ are proportional to the vacuum Higgs field which determines the electroweak unification scale. Therefore, the constants $m_{\mathrm{e}, \mathrm{q}} / \Lambda_{\mathrm{QCD}}$ can also be viewed as the ratio of the weak energy scale to the strong energy scale.

How can a space-time variation of the fundamental constants and a dependence on the gravitational potential occur? Light scalar fields very naturally appear in modern cosmological models, affecting $\alpha$ and $m_{\mathrm{e}, \mathrm{q}} / \Lambda_{\mathrm{QCD}}$. One of these scalar fields is the famous "dark energy" which causes accelerated expansion of the Universe. Another hypothetical scalar field is the dilaton, which appears in string theories together with the graviton. Cosmological variation of these scalar fields should occur because of drastic changes of the matter composition of the Universe. During the Big Bang nucleosynthesis the Universe was dominated by radiation, then by cold dark matter and now by dark energy. Changes of the cosmic scalar field $\varphi_{0}(t)$ lead to the variation of the fundamental constants $X\left(\varphi_{0}\right)$. Massive bodies (galaxies, stars, planets) can also affect physical constants. They have a large scalar charge $S=s_{p} Z+s_{e} Z+s_{n} N$, where $Z$ is the number of protons and electrons and $N$ is the number of neutrons; $s_{p}, s_{e}, s_{n}$ are the scalar charges of the proton, electron, and neutron, respectively. There is also a contribution of the nuclear binding energy (scalar charge of virtual mesons mediating nuclear forces).

The scalar charge produces a Coulomb-like scalar field $\varphi_{s}=S / R$, where $R$ is the distance from the charge. The total scalar field is $\varphi=\varphi_{0}+\varphi_{s}$, therefore 
we may have a variation of the fundamental constants inversely proportional to the distance from massive bodies,

$$
\begin{aligned}
X(\varphi) & =X\left(\varphi_{0}+\varphi_{s}\right)=X\left(\varphi_{0}\right)+\delta X(R), \\
\delta X(R) & =(d X / d \varphi) S / R,
\end{aligned}
$$

where a nonzero $\delta X$ violates LPI and a small $\varphi_{s}$ was assumed.

The gravitational potential $U$ is essentially proportional to the number of baryons $Z+N$ and inversely proportional to the distance $R, U=-G M / R$. Therefore, the change of the fundamental constants near massive bodies can be written:

$$
\delta X / X=K(X, i) \delta\left(U_{i} / c^{2}\right)
$$

where $i$ refers to a particular composition of the mass $M$, and where the coefficients $K(X, i)=-(d X / d \varphi) c^{2} S_{i} / G M_{i}$ are not universal, but are expected to be different for, e.g., the Sun and the Earth, $K(X$, Earth $) \neq K(X$, Sun $)$. Indeed, the Sun consists mostly of hydrogen; therefore the Sun's scalar charge is $S_{\text {Sun }} \simeq Z\left(s_{p}+s_{e}\right)$. The Earth contains heavier elements where the number of neutrons exceeds the number of protons, $N \simeq 1.1 Z$. Therefore, the scalar charge of the Earth $S_{\text {Earth }} \simeq Z\left(1.1 s_{n}+s_{p}+s_{e}\right)$ and is sensitive to the neutron scalar charge (and also to the contribution of the nuclear binding), in contrast to the Sun.

The most sensitive experiments suitable for a search of a space-time dependence in local or near-local experiments (such as satellite experiments) are clock-clock comparisons. In one type of terrestrial local experiment, dissimilar clocks located nearby are compared for a duration spanning at least one year. During such periods, the earth moves periodically closer and further away from the sun, so that the solar gravitational potential on Earth is modulated. This enables a search for a dependence of the fundamental constants on the sun's scalar charge $[17,18]$. These experiments are able to set limits to $K(X$, Sun) for $X=\alpha, m_{\mathrm{e}, \mathrm{q}} / \Lambda_{\mathrm{QCD}}$, at the level of $10^{-6}[12,13]$.

The proposed EGE satellite experiments are complementary to terrestrial experiments, allowing to set limits to $K(X$, Earth) and, combining with the results on $K(X$, Sun $)$ from terrestrial comparisons, to set limits on the dependence of the fundamental constants on the neutron scalar charge $s_{n}$.

In summary, when the frequencies of two identical clocks (of nominal frequency $v_{a}$ ) that are located at different positions $x_{1}, x_{2}$, are compared by measuring their frequency ratio at some position $x$, the result may be written as

$$
v_{a, 2}\left(x^{\prime}\right) / v_{a, 1}\left(x^{\prime}\right)=1+\left[1+\sum_{X} A_{X, a} K(X, i)\right]\left(U_{i}\left(x_{2}\right)-U_{i}\left(x_{1}\right)\right) / c^{2} .
$$

The sensitivity factor $A_{X, a}=X\left(\mathrm{~d}\left(\ln v_{a}\right) / \mathrm{d} X\right)$ is the sensitivity of the clock frequency to a particular constant $X$, and can be calculated using atomic and 
nuclear theory (see e.g. $[12,13])$. The measurement of this ratio, which is sensitive to $K$, represents a test of General Relativity's redshift.

When the frequencies of two co-located but dissimilar clocks a, b (of nominal frequencies $v_{a, 0}$ and $\left.v_{b, 0}\right)$ are compared in-situ at two locations $x_{1}, x_{2}$ (LPI test), the result is

$$
\begin{aligned}
& {\left[v_{a, 1}\left(x_{1}\right) / v_{b, 1}\left(x_{1}\right)\right] /\left[v_{a, 2}\left(x_{2}\right) / v_{b, 2}\left(x_{2}\right)\right]=1+} \\
& \quad\left[\Sigma_{X}\left(A_{X, a}-A_{X, b}\right) K(X, i)\right]\left(U_{i}\left(x_{2}\right)-U_{i}\left(x_{1}\right)\right) / c^{2} .
\end{aligned}
$$

The result of the EGE mission may be given as a limits for (or nonzero values of) $K(X$, Earth $)$, for the three fundamental constants $X=\alpha, m_{\mathrm{e}} / \Lambda_{\mathrm{QCD}}$, and $m_{\mathrm{q}} / \Lambda_{\mathrm{QCD}}$.

\subsection{Measurement approaches for the gravitational frequency shift}

The gravitational potential experienced by the ground and satellite clocks includes the contributions from the earth and the sun (the contribution from the moon is an order smaller and may be neglected for the purposes of the discussion, but will be taken into account in data analysis).

Nonzero gravitational frequency shift signals (Eq. 3) can be obtained in three ways. Method (1) below is the most sensitive, and represents a strong reason to employ an optical clock, which on short averaging times is able to provide a higher frequency stability than a microwave clock. Method (2) and (3) are less accurate, but are complementary in terms of measurement approach. Finally, method (3) addresses the solar gravitational frequency shift while (1) and (2) measure the terrestrial effect. The performance of the instruments assumed in the following is shown in Fig. 2.

(1) Repeated measurements of satellite clock frequency variation between apogee and perigee

In this measurement approach, the difference (or ratio) between earthsatellite clock frequency ratio at perigee and at apogee is considered. This difference is measured at each perigee and apogee passage, and averaged over the mission duration. Systematic shifts (if not correlated with orbital motion) are expected to average out, leading to a gain in sensitivity of up to $n^{1 / 2}$, where $n=$ number of orbits. This mode uses the stability properties of the satellite clocks on the timescale from perigee to apogee, rather than their accuracy. Assuming a specification of $\leq 3 \times 10^{-16}$ satellite optical clock fractional frequency instability for integration times between 1,000 and $25,000 \mathrm{~s}, n \cong 1,000$ comparisons (i.e. most orbits of the 3-year mission), and a perigee-apogee potential difference $(U$ (apogee $)-U($ perigee $)) / c^{2}=\Delta U / c^{2} \cong 4 \times 10^{-10}$, allows a 


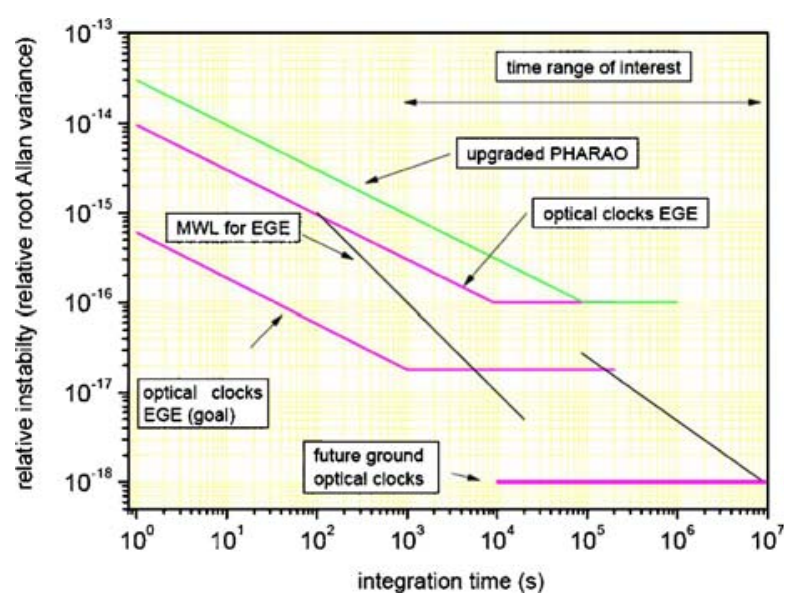

Fig. 2 Performance of clocks and links on EGE. Curve "optical clocks EGE" is the specification. Curve "optical clocks EGE (goal)" refers to expected near-future performance of an optical clock based on neutral atoms. "Upgraded PHARAO" refers to the PHARAO microwave clock using the high-quality microwave-optical local oscillator. The microwave link (MWL) performance exhibits a discontinuity since common-view contact time of ground stations with EGE is limited to several $\mathrm{h}$. The different slopes arise because on short time scales only white phase noise is relevant, while on long time scales, flicker noise also contributes. The reproducibility is taken into account

measurement of the redshift at $25 \mathrm{ppb}$ accuracy. The ground clocks to which the satellite clocks are compared to at perigee and at apogee will be located in different ground stations. The inaccuracy of these ground clocks must therefore be sufficiently smaller than $1 \times 10^{-17}$ in order not to contribute to the $25 \mathrm{ppb}$ error. Current state-of-the art inaccuracy is $2 \times 10^{-17}$ and is likely to decrease further.

(2) Absolute comparison between ground and satellite clocks with the satellite at apogee

Here the terrestrial gravitational potential difference is maximum, $U$ (apogee) $-U$ (perigee) $/ c^{2} \cong 6.5 \times 10^{-10}$. Assuming a space optical clock inaccuracy of $1 \times 10^{-16}$ and a ground clock inaccuracy a factor 3 lower (current state-of-the-art), allows a measurement of the redshift at $150 \mathrm{ppb}$. This measurement takes advantage of the repetitive nature of the orbit only to a small extent and relies on the accuracy of the optical clocks, in particular of the space clocks. It is thus complementary to (1), albeit less accurate.

In (1) and (2), the dominant contribution to the redshift is from Earth's gravitational potential. The contribution from the solar potential is strongly reduced due to the fact that satellite and earth are an interacting system in free-fall toward the Sun [19], and can be modeled.

(3) Comparisons between terrestrial clocks

The frequency comparison link to the EGE satellite allows terrestrial clock comparisons by a common-view method, whereby the frequency 
signals from two distant ground clocks are sent to the satellite and compared there. The frequency difference between two identical ground clocks is determined, if systematic instrumental errors are negligible, by the difference in gravitational potential at the two clock locations. This potential difference includes a terrestrial and a solar contribution.

The solar contribution varies according to the orientation of the clock pair with respect to the Sun with a 1 day-period. For two clocks at a surface distance of $\sim 6,000 \mathrm{~km}$ and suitable orientation with respect to the sun, the normalized modulation amplitude is $\cong 4 \times 10^{-13}$. This is the same as the earth potential difference for $4 \mathrm{~km}$ height difference. It is possible that ground clocks will have reached an inaccuracy at the level of $1 \times 10^{-18}$ at the time of the mission in 2015. Using the microwave link, a comparison of ground clocks over $\sim 3 \mathrm{~h}$ (typical common-view duration) with a resolution of $1 \times 10^{-17}$ can be performed, limited by the instability of the link. Repeating the clock comparison at each common-view window ( $n=1,000$ comparisons) will allow averaging, and a measurement of the solar gravitational shift with fractional inaccuracy of $1 \mathrm{ppm}$. In comparison, the best current results for the solar gravitational frequency shift are at the few-percent level [20,21]. A moderate averaging out of ground clock systematic shifts is assumed, as well as modeling of the earth gravitational potential variations on the daily timescale at the appropriate level.

(4) Comparisons of on-board clocks

The two on-board clocks are continuously compared; these data are analyzed for violation of LPI, (Eq. 4). Since the measurement is a null test, the required accuracy of the gravitational potential at the satellite location is low and easily satisfied.

\subsection{Test of local position invariance and search for neutron scalar charge}

The test of LPI is performed in conjunction with the measurement of the gravitational frequency shift.

With approach 2.3 (1), the optical clock will be able to measure $K$ ( $\alpha$, Earth) with accuracy $30 \mathrm{ppb}$ (spec).

Combining the results of the microwave and optical clocks will determine $K\left(m_{\mathrm{e}} / \Lambda_{\mathrm{QCD}}\right.$, Earth $)+\varepsilon K\left(m_{\mathrm{q}} / \Lambda_{\mathrm{QCD}}\right.$, Earth $)$ with accuracy $85 \mathrm{ppb}(\mathrm{spec})$, where $\varepsilon \approx-0.1$.

Simultaneously, the on-board comparison 2.3. (4) yields the combination $1.9 K(\alpha$, Earth $)+K\left(m_{\mathrm{e}} / \Lambda_{\mathrm{QCD}}\right.$, Earth $)+\varepsilon K\left(m_{\mathrm{q}} / \Lambda_{\mathrm{QCD}}\right.$, Earth $)$ with accuracy $85 \mathrm{ppb}$ (spec).

Note that these measurements are fully complementary to current and future terrestrial clock-clock comparisons, which are sensitive to $K(X, \operatorname{Sun})$, and can be combined with the latter to determine or set limits to the neutron scalar charge. 
2.5 Theoretical analysis of frequency comparison signals

The on-board clock-clock comparison signals can be analyzed straightforwardly in terms of a violation of LPI, since signal propagation effects are absent.

The determination of the gravitational frequency dilation via groundsatellite clock comparisons is more complex (Eq. 1 is strongly simplified) and requires an accurate treatment of all relevant relativistic effects. Gravitation in the solar system is described in the barycentric (BCRS) and geocentric (GCRS) non-rotating celestial reference systems by means of post-Newtonian solutions of Einstein's equations for the metric tensor in harmonic gauges codified in the conventions IAU2000 [22]. In these conventions the relativistic structure of Newton potential (order $1 / c^{2}$ ) and gravitomagnetic (order $1 / c^{3}$ ) potentials are given. For the Newton potential the multipolar expansion of the geopotential determined by gravity mapping is used. The post-Newtonian effects of the metric (described in terms of dimensionless parameters $\beta$ and $\gamma$ ) can be included. Orbits of satellites are evaluated in the GCRS at the level of a few $\mathrm{cm}$ by means of the Einstein-Infeld-Hoffmann equations at the order $1 / c^{2}$ [23] using the relativistic Newton potential. Instead the trajectories of the ground clocks in the GCRS are evaluated from their positions fixed on the Earth crust (ITRS, International Terrestrial Reference System) by using the non-relativistic IERS2003 conventions [24, 25].

For the propagation of light rays (here: radio signals) between the satellite and the ground stations one uses the null geodesics of the post-Newtonian solution in the GCRS. The time/frequency transfer properties have been theoretically evaluated for an axisymmetric rotating body [26-29] at the order $1 / c^{4}$ (i.e. with Newton and gravitomagnetic potentials developed in multipolar expansions). These results have been developed for use in the ACES mission. The relative frequency dilation can be expressed as

$$
\frac{\Delta v}{v}=1+\frac{1}{c} L_{1}+\sum_{n=2}^{4} \frac{1}{c^{n}}\left(L_{n}+G_{n}\right)
$$

where $L_{n}$ describes special-relativistic Doppler effects and $G_{n}$ the generalrelativistic effects. These quantities are time-dependent due to the orbital motion. For concreteness, we report the orders of the terms. The kinematical effects are $\left|L_{1} / \mathrm{c}\right|<1 \times 10^{-5},\left|L_{2} / \mathrm{c}^{2}\right|<2 \times 10^{-10},\left|L_{3} / \mathrm{c}^{3}\right|<3 \times 10^{-15},\left|L_{4} / \mathrm{c}^{4}\right|<$ $7 \times 10^{-20}$. The Newtonian contributions are $G_{2}=G_{2}^{(M)}+G_{2}^{\left(J_{2}\right)}+G_{2}^{\left(J_{4}\right)}+G_{2}^{\left(J_{6}\right)}$ ( $M$ is the mass monopole, $J_{n}$ are the lowest multipoles), with estimates $\left|G_{2}^{(M)} / \mathrm{c}^{2}\right|<3 \times 10^{-11},\left|G_{2}^{\left(J_{2}\right)} / \mathrm{c}^{2}\right|<2 \times 10^{-13},\left|G_{2}^{\left(J_{4}\right)} / \mathrm{c}^{2}\right|<3 \times 10^{-16},\left|G_{2}^{\left(J_{6}\right)} / \mathrm{c}^{2}\right|<$ $1 \times 10^{-16}$. For $G_{3}=G_{3}^{(M)}+G_{3}^{\left(J_{2}\right)}$, the estimates are $\left|G_{3}^{(M)} / \mathrm{c}^{3}\right|<2 \times 10^{-14}$, $\left|G_{3}^{\left(J_{2}\right)} / \mathrm{c}^{3}\right|<1 \times 10^{-18}$. Finally, the $\mathrm{G}_{4}=\mathrm{G}_{4}^{(M)}+\mathrm{G}_{4}^{(S)}$ contributions $\left(\mathrm{G}_{4}^{(S)}\right.$ is the lowest gravitomagnetic effect) are of order $\left|\mathrm{G}_{4}^{(M)} / \mathrm{c}^{4}\right|<1 \times 10^{-19},\left|\mathrm{G}_{4}^{(S)} / \mathrm{c}^{4}\right|<$ $1 \times 10^{-22}$, and thus not relevant for EGE. 
The available theory is fairly complete for the data analysis in mode 2.3 (1), described below. For modes $2.3(2,3)$, the theory will need to be generalized to include the effect of the gravitational potential at the location of the ground clock(s) (including time-varying effects, such as the tides) and of the solar and lunar potentials.

The uncertainties of the various contributions above are minimized by a using precise EGE orbit data obtained by satellite tracking, and by using current and future accurate earth gravity information. Required orbit position knowledge is at $1 \mathrm{~cm}$ level near perigee, and $\sim 50 \mathrm{~cm}$ near apogee, reachable already today with the proposed orbitography approach described below. The validity of the special-relativistic Doppler shift has been and will continue to be independently verified with increased accuracy by spectroscopy of relativistic atomic ion beams [30]. Potential violations of this aspect of Lorentz Invariance are expected to be sufficiently bounded so as not to affect the signal interpretation.

\subsection{Local Lorentz invariance tests}

There has been an explosion of interest in Local Lorentz invariance in recent years [31, 32], with numerous astronomical studies and high-precision experiments applied to search for violations of Lorentz invariance. Significant developments have also occurred on the theoretical side, and a theory (standard model extension, SME [33]) has been worked out that provides a unified framework for describing and analyzing Lorentz Invariance violations in a variety of systems. Two aspects of Lorentz Invariance can be tested with EGE.

\subsubsection{Independence of the speed of light from the laboratory velocity}

A possible dependence of the speed of light on the speed $v$ of the laboratory can be modeled, according to the Mansouri-Sexl test theory [34], by

$$
c(v)=c_{0}\left(1+B v^{2} / c^{2}\right)
$$

Here $v \simeq 377 \mathrm{~km} / \mathrm{s}$ is the velocity with respect to the cosmic microwave background, the cosmologically preferred frame, and $B$ is a combination of parameters describing deviations from the usual Lorentz transformation formulas. $B=0$ if Lorentz Invariance holds. For a terrestrial experiment, the rotation of the Earth around its axis modulates $v$ with a $300 \mathrm{~m} / \mathrm{s}$ amplitude. A dependence $c(v)$ can be searched for by measuring the frequency difference between a resonance frequency of a highly stable optical cavity (cavity frequencies are proportional to $c$ ) and an optical clock (Kennedy-Thorndike-type experiment [35]) and determining the modulation of the frequency difference correlated with the modulation of $v$. The advantages of a space experiment are the high orbital velocity and strongly reduced cavity deformation thanks to microgravity [36, 37]. On the proposed elliptic orbit, $v$ varies between +4 and $-4 \mathrm{~km} / \mathrm{s}$ over approximately $1 \mathrm{~h}$. This variation is 13 times larger 
than for the case of a terrestrial experiment. The shorter time scale is also advantageous, since the drift of the cavity is more predictable. In the EGE mission, the reference cavity of the clock laser is used. The large number of orbits improves the statistics. An improvement of the accuracy of $B$ by a factor 20 compared to the best current terrestrial results is realistic. The data analysis can also be performed in the framework of the SME theory, taking into account complementary bounds obtained from terrestrial experiments and astrophysical observations.

\subsubsection{Independence of Zeeman splitting frequency on the direction of the magnetic field}

One class of tests of Lorentz Invariance consists in measuring the frequency splitting between two levels of a quantum system induced by a static magnetic field, as a function of orientation of the field direction with respect to the stars (Hughes-Drever-type experiments [31]). This class addresses the socalled matter sector, and is complementary to combined-photon/matter-sector tests such as the above. The most precise experiments are performed with atomic clocks, e.g. masers or cold atom clocks [38]. The EGE instruments allow such experiments, since magnetic fields are applied continuously in the clock or repeatedly for calibration. Compared to terrestrial experiments, the high orbital velocity of EGE could lead to an improvement by a factor $\sim 20$.

\subsection{Application to geophysics}

Using GPS, coordinates of a point on the Earth can be obtained with an inaccuracy well below $1 \mathrm{~cm}$ in a well defined international terrestrial reference system. The coordinates are purely geometrical and do not contain any gravity information. A local gravitational potential $U_{B}$ is determined with respect to a reference gravitational potential $U_{A}$ by "levelling" the surface of the Earth, i.e. sequentially measuring gravity $\vec{g}$ and height differences every ca. $50 \mathrm{~m}$ :

$$
U_{B}-U_{A}=-\int_{A}^{B} \vec{g}(\vec{r}) \cdot d \vec{n},
$$

where $\vec{n}$ is the difference vector between subsequent locations. The disadvantage of measuring height differences by the levelling method is a random walk effect of accumulated errors.

In the classical definition, a geoid is defined as the particular equipotential surface nearest to mean sea level. In these terms, the geoid serves as a reference surface for measuring the height and also to define a reference for the gravitational potential. Such a vertical reference frame, historically was realized for a country or several countries by determining the mean of sea level observations at tide gauge stations taken over long period of time. However, modern satellite altimetry missions such as TOPEX/POSEIDON or ENVISAT show that departure of the mean sea level from an equipotential 
surface may reach up to several meters on the global scale, see e.g. [39]. Therefore, height systems based on different tide gauge stations may differ in the realisation of the geoid and may differ with respect to the reference by several meters.

The best gravity field models obtained from satellite data (e.g. mission GRACE) have reached a precision of about $1 \mathrm{~cm}$ over $\sim 250 \mathrm{~km}$ half wavelength [40]. Satellite data also reveals changes in time [41]. However, for typical Earth topography with height variations of e.g. 1,000 m over $30 \mathrm{~km}$ horizontal distance, one may expect variations in the geoid of about $80 \mathrm{~cm}$. Such a high-spatial-frequency signal in geoid variations cannot be detected by space gravity missions and requires a combination of satellite and terrestrial gravity measurements, like gravity anomalies, deflections of vertical and GPS/levelling points. The best combined global gravity field models are provided up to degree and order 360 in terms of spherical harmonic representation, corresponding to a half-wavelength of about $55 \mathrm{~km}$. However, in combining the satellite and terrestrial gravity field measurements the problem of terrestrial data given in different height systems between continents and different countries remains and has to be tied to satellite measurements.

The geodetic scientific community is currently establishing a Global Geodetic Observing System (GGOS), [42]. Its objectives are the early detection of natural hazards, the measurement of temporal changes of land, ice and ocean surfaces as well as the monitoring of mass transport processes in the Earth system. Global change processes are small and therefore difficult to quantify. Therefore the required precision, relative to the Earth's dimension is $1 \mathrm{ppb}$. GGOS will be established by the combination of geodetic space techniques (GPS, Laser-Ranging, very large baseline interferometry) and realized by a very large number of terrestrial and space-borne observatories. The purely geometric terrestrial 3D coordinate system is in good shape and fully operational. It has to be complemented, however, with a globally uniform height system of similar precision. The current precision level of regional height systems, in terms of gravity potential differences, is in the order of $1 \mathrm{~m}^{2} / \mathrm{s}^{2}(10 \mathrm{~cm})$ with inconsistencies between these various systems up to several $10 \mathrm{~m}^{2} / \mathrm{s}^{2}$ (several meters). The actual requirement in the context of GGOS is $0.1 \mathrm{~m}^{2} / \mathrm{s}^{2}(1 \mathrm{~cm})$ with the need of a permanent, i.e. dynamical, control. This requirement of high precision height control comes from the need to understand, on a global scale, processes such as sea level change, global and coastal dynamics of ocean circulation, ice melting, glacial isostatic adjustment and land subsidence as well the interaction of these processes. Only by means of monitoring in terms of gravity potential changes at the above level of precision the change of ocean level can be understood as a global phenomenon and purely geometric height changes be complemented by information about the associated density or mass changes.

The geoid can also be defined in a relativistic way [43], as the surface where accurate clocks run with the same rate and where the surface is nearest to mean sea level. The relation between the differences in the clock frequencies and the gravitational potential is given in simplified form by Eq. 1. At present 
there is no operational way to compare frequencies of the already available optical clocks on the global scale at the same level as their accuracy would allow. With EGE and by then improved ground optical clocks it will become possible to obtain gravitational potential differences on a global scale by comparing frequencies. This would be a significant new dimension to gravity field determination, since such observables are given on the global scale and provide in situ local gravity information at the same time. EGE will allow clock-based gravitational potential mapping with the same payload instruments used for the fundamental physics experiments. The on-board clocks are actually not required for this purpose, MOLO, MWL and FCDP suffice. Thus, EGE will allow establishing a global reference frame for the Earth gravitational field with accuracy in the order of a few $\mathrm{cm}$ in terms of the geoid heights. This assumes that by the time EGE is operational, (mobile) ground clocks with fractional inaccuracy at the level of $10^{-18}$ are available; the measurements are limited by MWL noise and thus require a long integration times to match the expected ground clock performance. Such a reference frame will serve as reference for all gravity field modeling and clocks will be used to define the international atomic time scale and the Earth gravitational potential at the same time.

In summary, with optical clocks geodesy will undergo a second revolution: after geometry now being measured by clock-based GNSS systems, also physical heights and potential will be measured by (mobile) optical clocks using the gravitational redshift effect.

\subsection{Application to frequency standards and terrestrial fundamental physics studies}

As has been described, repeating ground clock comparisons and integrating over several months will provide global ground clock frequency comparisons at the level of $1 \times 10^{-18}$, more than two orders of magnitude below the current GPS and two-way satellite time and frequency transfer methods. In the time and frequency community, the availability of such a global high performance microwave link will accelerate the process leading to a redefinition of the SI second based on clocks operating in the optical domain.

The global character of the ground clock comparisons made possible by EGE can also contribute to the search for a time-variation of the fundamental constants.

The comparisons do not require availability of the on-board clocks. The risk of this type of measurements is therefore reduced. Moreover, the lifetime of many subunits required for this measurement (MWL and FCDP, see below) can exceed 10 years (from previous experience with similar devices) and thus offers a motivation to increase the mission duration beyond the nominal 3 years.

Further applications to metrology are listed under the secondary goals in Section 2.1 above. 


\section{Mission profile}

\subsection{Orbit}

The orbit must satisfy the following requirements:

Primary: 1. Large gravitational potential difference between apogee and perigee,

2. sufficient contact time with at least two ground stations at perigee

Secondary: Simultaneous visibility of satellite from distant (intercontinental) ground stations for several hours.

To satisfy the primary objective, the orbit must be highly elliptic. The perigee should be low. A very low perigee means a short contact time, which decreases the accuracy of the frequency measurement. A compromise is a perigee altitude of $\sim 2,500 \mathrm{~km}$. For a sufficiently high apogee, this yields a $\Delta U$ of $60 \%$ of the theoretical maximum between the earth surface and infinity.

The baseline scenario is an orbit of approx. $12 \mathrm{~h}$ period, with the following properties:

Apogee Altitude $37856 \mathrm{~km}$ (-10 deg latitude, one over Atlantic and Pacific, resp.)

Perigee Altitude $2500 \mathrm{~km}(+10$ deg latitude, one over Guatemala and Malaysia, resp.)

Inclination $\quad 63.4^{\circ}$, argument of perigee: $170^{\circ}$

RAAN $25^{\circ}$, True Anomaly: $220^{\circ}$

Apogee drift rate $70 \mathrm{~km} /$ day, perigee drift rate $\sim 14 \mathrm{~km} /$ day

True anomaly and right ascension of the ascending node (RAAN) were optimized to minimize orbital precession. Figure 3 shows the ground track and the ground coverage at one of the perigees. Common-view contact time for stations in Europe is about $6 \mathrm{~h}$, while between a station in Europe and in the USA it is about $3.5 \mathrm{~h}$. Frequency comparison windows between a given ground station and EGE repeat every $24 \mathrm{~h}$.

A final selection of the orbit requires an in-depth study of the costs of the propellant and maneuvers as well as of the setting up of new ground stations.

\subsection{Ground segment}

The mission requires two separate ground segments: the science ground segment and the standard satellite control segment which supports telemetry and telecommand (TM/TC). 


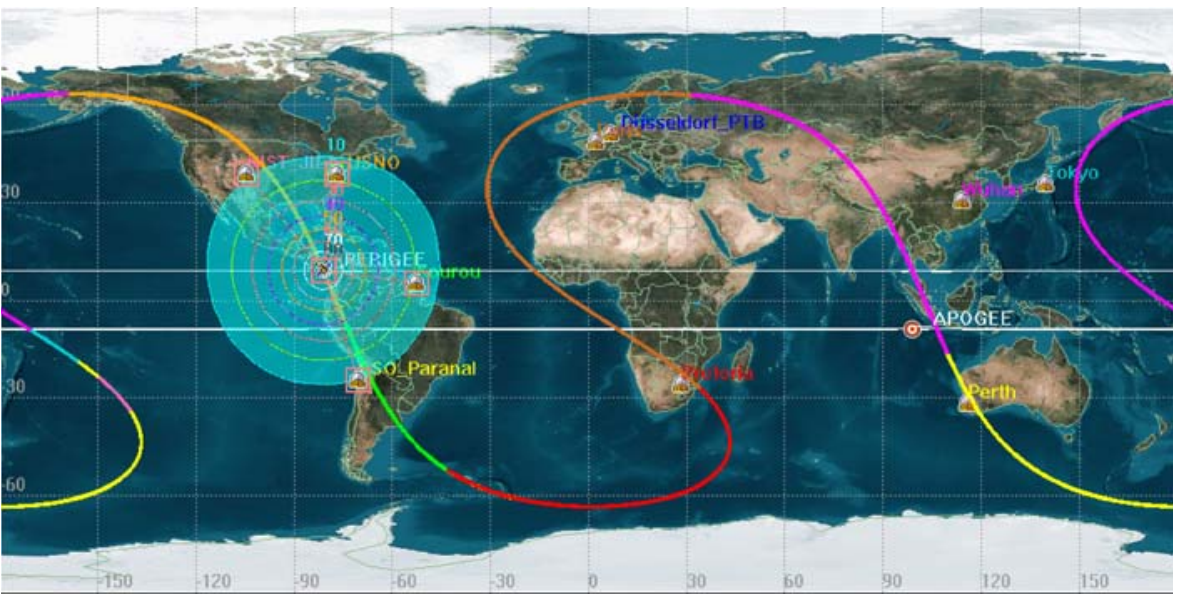

Fig. 3 Baseline EGE orbit. One (out of two) apogee and one (out of two) perigee ground points are shown. The ground coverage at one of the perigees is shown, with circles at elevation angle steps of $10^{\circ}$, starting from $10^{\circ}$

\subsubsection{Science ground segment}

As described above, one part of the mission is to perform frequency comparisons between ground-to-space and ground-to-ground (common-view and non-common view). The use of a two-way microwave link (MWL) system has the advantage that a precise orbit or the precise relative velocity of the satellite must not be known [4]. The orbit determination requirements for a two-way system are significantly relaxed compared to the requirements to model the relativistic effects on the clocks. The frequency comparison results are available both on ground and on the satellite.

For ground-to-satellite clock comparisons five ground stations are favourable, two capable of viewing perigee, three for viewing apogee. This allows for the classic three-clock configuration, where the performance of each clock can be obtained individually. The perigee stations will be observing the satellite for $\sim 25$ min every perigee passage for the duration of the mission. The apogee stations will observe for $\sim 3 \mathrm{~h}$ every apogee passage. Of course, a larger number of stations, as well as longer observation time are useful for robustness, redundancy, and orbit determination. The stations could be installed on a temporary basis for the duration of the mission.

The stations must have access to optical clocks of performance at least as high as today's state-of-the-art ground clocks, but preferably higher. They will include an optical frequency comb, by means of which the optical clock frequency is transformed to a radio frequency for comparison with the EGE clocks. High-accuracy fiber-optic link technology already existing today allows distances of $\sim 100 \mathrm{~km}$ between the ground station and the corresponding optical clock, allowing a certain freedom in the choice of the clock location. With further increase of the fiber-optic link range, currently being investigated, 
the area over which the ground station can be positioned relative to the optical clock will increase. This option is of importance for the implementation of a geophysics measurement campaign for which a large number of clock locations is of interest.

The above ground stations will be complemented by some 15 additional ground terminals provided by the world-wide laboratories equipped with high performance primary clocks at the time of the mission. These will participate in orbit determination to $1 \mathrm{~cm}$ accuracy along the full orbit, which can be achieved with non-optical atomic clocks. The EGE MWL is fully compatible to planned ACES ground terminals, which might reduce the number of required stations.

With a larger number of transportable optical clock ground stations deployed and moved across the continents, a geophysics campaign could be implemented to map the gravitational potential.

Ground station equipment MWL uses dedicated small transportable terminals, which can be easily installed at any location of interest. Each terminal consists of a steerable dish antenna of $1 \mathrm{~m}$ diameter, with pointing accuracy of $0.1^{\circ}$, using a transmitted power of $5 \mathrm{~W}$ per band. The high directivity antenna (beam aperture Ka-band: $0.7^{\circ}$, Ku-band: $1.4^{\circ}$, S-band: $20^{\circ}$ ) avoids multi-path from nearby objects. Antenna noise temperatures are: Ka-band: $450 \mathrm{~K}, \mathrm{Ku}-$ band: $340 \mathrm{~K}$. Operation is at elevations above $10^{\circ}$ and measurements are performed above $20^{\circ}$. For ranging purposes, terminal location is determined by GPS surveying. Each ground station computes its own ionospheric corrections based on a triple-band receiver. The terminal has a built-in delay monitor, which allows calibrated ranging during several months, once its bias has been determined by laser ranging. Its $\mathrm{M}$ and $\mathrm{C}$ interface is via local area network to either a user's computer or directly to the science network control centre by remote M\&C. The station's operation is fully automatic and unattended. Schedule and orbital data are received via LAN once per day. Signal acquisition is fully autonomous. Data transmission is after each pass. The terminal has an uplink telecommand capability of $1 \mathrm{kBit} / \mathrm{s}$ and a downlink data capacity of up to $10 \mathrm{kBits} / \mathrm{s}$. For the local user, there is a visual interface, providing quicklook data from real-time comparison to the spacecraft clocks to verify their good health. The final full performance product is calculated at the science data centre.

Local metrological data (temperature, pressure, humidity) are recorded to correct for tropospheric delay of the ranging data. The main advantage of MWL terminals compared to laser based systems is their all-weather capability, although microwaves suffer higher propagation errors in the ionosphere and troposphere.

The EGE ground terminal will profit from the ERS-2 mission PRARE (precise range and range rate equipment) [45] experiments whose terminals had similar design and have been operated under extreme climatic conditions, incl. Antarctica and high temperature regions, from existing designs from the space segment (mission ACES), and no further special developments are deemed necessary. Cost per EGE master station is modest. 
Satellite tracking via laser ranging Laser ranging is alternative to MWL ranging, and also serves for absolute calibration of the latter. For this purpose an on-board corner cube reflector (CCR) is provided. Ranging will be done with the International Laser Ranging Service. Ideally, the satellite will be ranged at every perigee passage and every apogee passage, simultaneously with the frequency comparison procedures. Today's ranging precision is sufficient to satisfy the science goals. For apogee observations in the northern hemisphere, a large number of laser ranging stations is available. The number of stations capable of ranging at or near perigee in the southern hemisphere is six, and thus is sufficient, even considering unfavourable weather conditions at some of these. The MWL ground stations are able to range even under those conditions.

\subsubsection{Control ground segment}

Average data transfer rate is estimated at $300 \mathrm{MB} /$ day (approx. $40 \mathrm{kB} / \mathrm{s}$ on average). Data will be stored on board (2 GB capacity required) and will be transferred to a receiving station on the Earth once a day.

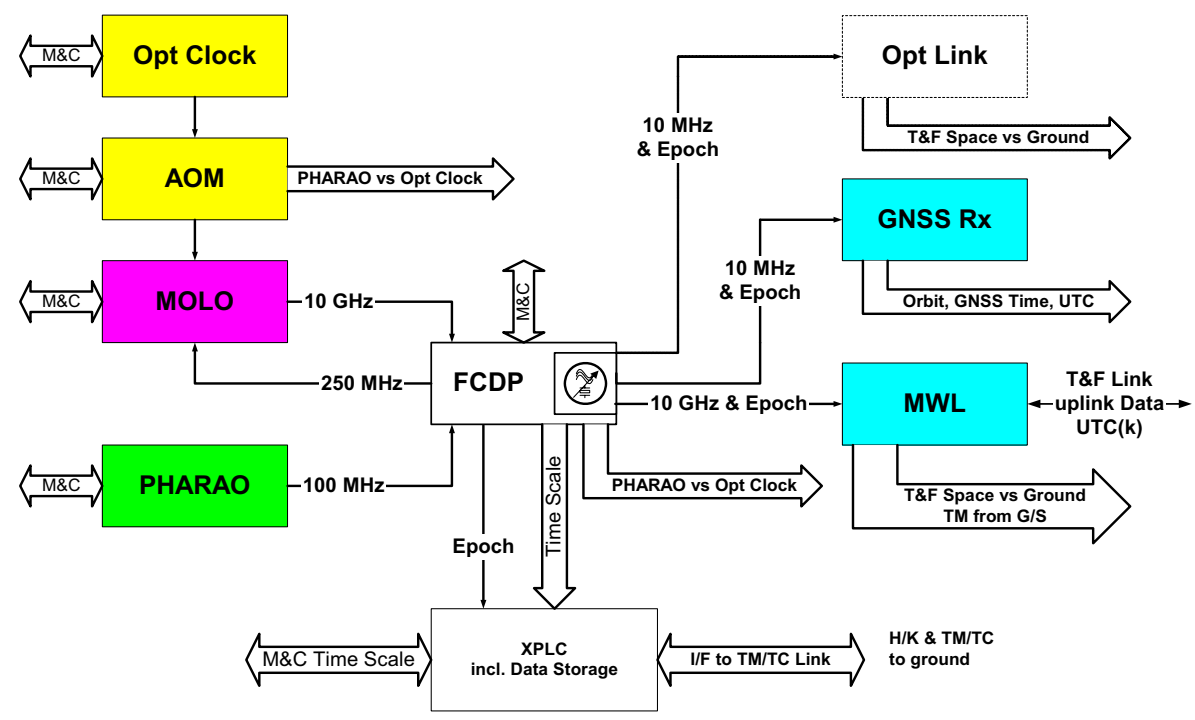

Fig. 4 Schematic of the payload showing subunits and their signals. The results of the frequency comparison between the two on-board clocks (optical clock and microwave clock PHARAO) are obtained from a combination of the frequency values applied to the frequency shifters AOM (see Figs. 7 and 8) and those output from the frequency control and distribution package (FCDP), as indicated by the arrows "PHARAO vs. Opt. Clock". The satellite clock to ground clock comparison results are output from the MWL. XPLC is the on-board computer, and is involved in generation of signals for instrument control. The optical link shown dashed is a possible add-on providing an alternative to the MWL. $G / S$ : ground stations. $M \& C$ : monitoring and control. $H / K$ : housekeeping. $T \& F$ : time and frequency 


\section{Main payload instruments}

\subsection{Overview}

The satellite payload consists of a pair of cold-atom clocks, a microwaveoptical local oscillator (MOLO), an on-board frequency distribution unit (FCDP), a satellite-to-ground clock comparison unit (MWL), and the onboard computer/control system (XPLC), see Fig. 4. Auxiliary units are the position determination GPS/Galileo receiver (GNSS Rx), attitude monitor (star tracker, not shown), and the corner cube reflector array (CCR, not shown).

The science goals require clocks of very high frequency stability on the timescales of half the orbital period $(6 \mathrm{~h})$ as well as perigee passage $(\sim 1,000 \mathrm{~s})$, see Fig. 2. Only clocks based on cold atoms are suited for the science goals. Table 1 reports values for a subset of atomic clocks that have been investigated, not including clocks that require cryogenic operation, or are too complex for use in an medium-class mission. The choice of a clock pair rather than a single one fulfils several requirements: it addresses more science goals, improves the validity of some science results, and it enables calibration, accuracy evaluation, and redundancy. The validity of the science results is enhanced, since the clocks differ not only in the employed atoms, but also in terms of having different sensitivity to perturbations by electric and magnetic fields, electronics errors, and misalignments. A comparison of the results arising from each clock allows for an understanding of measurement errors. Both the clocks' and the frequency comparison unit's performances will be determined after launch and regularly verified during the course of the mission. The only reliable

Table 1 Properties of different clock candidates for EGE

\begin{tabular}{llrllll}
\hline $\begin{array}{l}\text { Clock } \\
\text { atom }\end{array}$ & $\begin{array}{l}\text { Clock } \\
\text { frequency }\end{array}$ & $\begin{array}{l}\text { Fractional } \\
\text { inaccuracy }\end{array}$ & $\begin{array}{l}\sigma=\text { Fractional } \\
\text { instability } \\
\text { at } 1,000 \mathrm{~s}\end{array}$ & $\begin{array}{l}\text { Sensitivity } \\
\text { to } \alpha, A_{\alpha}\end{array}$ & $\begin{array}{l}\text { Figure of } \\
\text { merit }\left|A_{\alpha}\right| \\
\sigma\left[10^{16}\right]\end{array}$ & $\begin{array}{l}\text { Sensitivity } \\
\text { to } m_{\mathrm{e}, \mathrm{q}} /\end{array}$ \\
\hline $\mathrm{Sr}_{\mathrm{QCD}}$
\end{tabular}

A relevant figure of merit is the ratio of $\left|A_{X}\right|$ and fractional frequency instability $\sigma$ on the perigee visibility timescale. The Cs clock is the only high performance clock with adequate sensitivity to the particle masses $m_{\mathrm{e}}, m_{\mathrm{q}}$, The indicated Cs clock performance was achieved using a microwave local oscillator based on a cryogenic oscillator. On EGE, the latter is to be replaced by an optical local oscillator-based device (MOLO) with similar short-term performance, but without the need to operate at cryogenic temperature. The clock instability at $10,000 \mathrm{~s}$ is typically similar to the indicated inaccuracy

(p) projected, (d) demonstrated, (1) quadropole, (2) octupole 
way to achieve this is by having at least two clocks on board. The on-board clock-clock comparison provides performance evaluation independent of the ground-satellite comparison unit. Once the satellite clock performances are established, the link performance can be established as well.

The clocks are similar in basic structure, but differ in the detailed implementation. They consist of:

a source of atoms

a preparation subunit, which cools the atoms to near-standstill (millikelvin temperature or below)

a trapping subunit that confines the atom(s) to a tiny volume

an interrogation subunit

a clock control electronics package.

The clocks share a common, central unit, the optical-microwave local oscillator, which serves as a flywheel oscillator with outstanding stability on short timescales $(0.1-10 \mathrm{~s})$ for both clocks. It consists of a laser stabilized to an ultralow loss optical resonator made of ultra-low-expansion glass. A microwave signal is derived from the laser by phase-locking a frequency comb to the laser wave and using a multiple of the repetition rate.

While the subunits of any optical clock have a similar technological basis (optics, electrooptics, acoustooptics, thermal control, vacuum systems), they differ in their specifications. Depending on the choice of the atom species, delivery, confinement and interrogation methods, clocks of different performance result. A number of laboratory optical clock demonstrators have been developed worldwide. This ensures availability of the know-how for the industrial implementation of the flight models.

The first clock is a microwave clock based on the interrogation of slowly moving ensembles of cold Cs atoms. It is essentially based on the PHARAO clock [5, 6], developed to engineering model level by CNES in the framework of the ESA ISS mission ACES. In EGE, this clock is upgraded using the microwave-optical local oscillator. This improves significantly the clock performance. The specification is a relative instability (Allan deviation) of $3 \times 10^{-14}(\tau / \mathrm{s})^{-1 / 2}$, i.e. at the integration time $\tau=1,000 \mathrm{~s}$ it drops to $1 \times 10^{-15}$, and to $3 \times 10^{-16}$ at $10,000 \mathrm{~s}$. While this specification is at least a factor 3 less stringent than for the optical clocks, the significantly lower cost of obtaining a flight model and the sensitivity of its frequency to the particle masses makes it a suitable choice.

The optical clock baseline instrument is a single-ion optical clock. Among various suitable ion species $\left(\mathrm{Hg}^{+}, \mathrm{Al}^{+}, \mathrm{Sr}^{+}, \mathrm{Yb}^{+}\right)$[44-47], the $\mathrm{Yb}^{+}$ion has been selected. Its breadboard performance is established at the instability level of $3 \times 10^{-16}$ over $1000 \mathrm{~s}$, it exhibits a large sensitivity coefficient $A_{\alpha}$, has small sensitivity to magnetic fields, and exhibits significant potential for further improvements (use of the octupole transition, with $\sim 6$ times larger $A_{\alpha}$, and lower overall instability [48]; see below). A trapped $\mathrm{Yb}^{+}$ion is also a very robust system: it has been demonstrated that a single $\mathrm{Yb}^{+}$ion can remain trapped uninterruptedly for many months. Finally, $\mathrm{Yb}^{+}$is the only ion with 
which optical clocks demonstrators have been operated in not a single, but two major metrology laboratories,

Another attractive optical clock is based on ensembles of cold neutral $\mathrm{Sr}$ or $\mathrm{Yb}$ atoms trapped in an optical lattice produced by laser beams. This clock type has a lower instability than a single-ion clock thanks to the large number of atoms $\left(\sim 10^{5}\right)$ used. Its development is in progress in several laboratories in the world (in part supported by space agencies), and has to date reached $6 \times 10^{-17}$ instability at $5,000 \mathrm{~s}$ and $1 \times 10^{-16}$ uncertainty [49,50], with potential for even higher performance. However, it is more complex and costly than the $\mathrm{Yb}^{+}$ion clock, since the cold atom preparation and trapping subunits include more components, require higher laser powers, and the respective operating procedures are more complex. It is therefore not considered for the present mission designed to be developed in the very near future with limited resources, but is an interesting candidate for missions without such constraints. Its estimated physical parameters are indicated in Table 2 for comparison.

\subsection{Main instruments}

\subsubsection{Microwave clock}

The PHARAO cold atom clock is one of the two atomic clocks of the space mission ACES managed by ESA. It is proposed here to include a second version of this instrument as its cost/performance ratio is very attractive for the EGE scientific objectives. Its concept is very similar to ground based atomic fountains, but with the major difference of zero-g operation. Atoms slowly launched in free flight cross two microwave fields tuned to the transition between the two hyperfine levels of the cesium ground state. The interrogation method, based on two separate oscillating fields (Ramsey scheme), allows the detection of an atomic line whose width is inversely proportional to the transit time between the two microwave cavities. The resonant microwave field at $9.192631770 \mathrm{GHz}$ (SI definition of the second) is synthesized starting from an ultrastable quartz oscillator (USO) in the FCDP, phaselocked to the microwave output of the microwave-optical local oscillator and stabilized to the clock line using the error signal generated by the cesium resonator. In this way, the intrinsic qualities of the cesium hyperfine transition, both in terms of accuracy and frequency stability, are transferred to the USO. In a microgravity environment, the velocity of atoms along the ballistic trajectories is constant and can be changed continuously over almost two orders of magnitude (5$500 \mathrm{~cm} / \mathrm{s}$ ). Differently from atomic fountain clocks presently operated on ground, very long interaction times (up to few seconds) will be possible, while keeping reasonable the size of the instrument.

The instrument consists of 4 main elements, a laser source which provides the light required to cool and detect the cesium atoms (Fig. 5, left), a frequency chain which generates the $9.2 \mathrm{GHz}$ microwave signal, a cesium vacuum tube where the interaction between the microwave field and the atoms occurs 
Table 2 Resources and technology readiness level (TRL) of the EGE spacecraft

\begin{tabular}{|c|c|c|c|c|c|}
\hline Unit & $\begin{array}{l}\text { Volume } \\
{[1]}\end{array}$ & $\begin{array}{l}\text { Mass } \\
{[\mathrm{kg}]} \\
\end{array}$ & $\begin{array}{l}\text { Power } \\
{[\mathrm{W}]}\end{array}$ & TRL & Comment \\
\hline Ion clock & 100 & 70 & 60 & & \\
\hline Atom source & & & & 4 & \\
\hline Atom preparation & & & & 6 & \\
\hline Atom trap & & & & 4 & \\
\hline Atom interrogation & & & & 6 & \\
\hline Clock control & & & & 6 & \\
\hline Microwave clock & 200 & 91 & 110 & 8 & TRL by end 2008 \\
\hline \multicolumn{6}{|l|}{ Local oscillator } \\
\hline Stabilized laser & 18 & 15 & 23 & 6 & \\
\hline Frequency comb & 20 & 16 & 38 & 6 & \\
\hline Microwave link & 18 & 18 & 55 & 8 & \\
\hline FCDP & 7 & 8 & 12 & 8 & \\
\hline GPS receiver & 2 & 5 & 7 & 9 & \\
\hline CCR & 2 & 2 & 0 & 9 & \\
\hline Payload sum & 367 & 225 & 305 & & \\
\hline Thermal control & 14 & 17 & 30 & 8 & \\
\hline OBDH & 20 & 24 & 38 & 9 & \\
\hline Star tracker & 1 & 1 & 8 & 9 & \\
\hline Radiation shield & 10 & 40 & 0 & 9 & \\
\hline Support structure & 170 & 204 & 0 & 9 & \\
\hline Power incl solar arr. & 50 & 58 & 10 & 9 & \\
\hline Propulsion & 21 & 25 & 20 & 9 & \\
\hline AOCS & 36 & 43 & 82 & 9 & \\
\hline $\mathrm{TM} / \mathrm{TC}$ & 8 & 10 & 15 & 9 & \\
\hline Satellite bus sum & 330 & 422 & 203 & & Flexbus concept \\
\hline Payload plus bus incl. $20 \%$ margin & 836.4 & 776.4 & 609.6 & & \\
\hline Propellant & & 40 & & & \\
\hline Launch adapter & & 100 & & & \\
\hline $\mathrm{S} / \mathrm{C}$ wet mass/power incl. Margins & & 916.4 & & & \\
\hline Lattice clock & 135 & 105 & 152 & & Alternative clock \\
\hline Atom source & & & & 4 & \\
\hline Atom preparation & & & & 6 & \\
\hline Atom trap & & & & 4 & \\
\hline Atom interrogation & & & & 6 & \\
\hline Clock control & & & & 6 & \\
\hline
\end{tabular}

Also included are the resources for an optical lattice clock based on neutral Strontium atoms

(Fig. 5, right), and a computer. The engineering model of PHARAO is fully assembled at CNES Toulouse and undergoes functional and performance tests until the end of 2008 . The operating temperature range is $10-33^{\circ}$ and non-operating temperatures are $-45^{\circ},+60^{\circ}$. The cesium tube consists in a UHV chamber pumped by getters and a $3 \mathrm{l} / \mathrm{s}$ ion pump, a cesium reservoir, a microwave cavity, coils to provide a uniform magnetic field, and three layers of magnetic shields. Vacuum windows with fiber-optics collimators enable the transmission of the cooling and detection light onto the cesium atoms.

In autonomous mode, PHARAO uses the USO as interrogation oscillator and will provide a clock signal with fractional frequency stability below $1 \times 10^{-13}(\tau / \mathrm{s})^{-1 / 2}$, and inaccuracy near $1 \times 10^{-16}$. When using the microwave- 

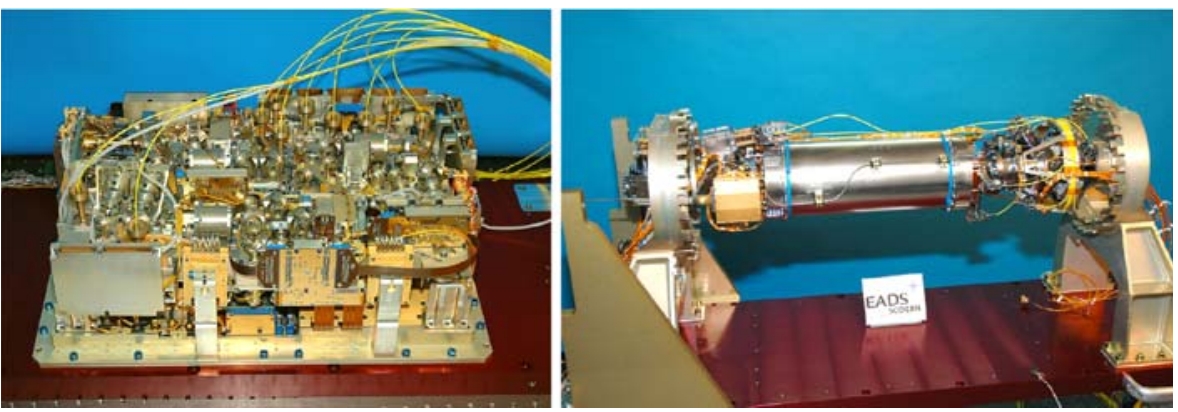

Fig. 5 Left: The PHARAO laser source engineering model with cover removed. Dimensions are $530 \times 350 \times 150 \mathrm{~mm}^{3}$ and the mass is $20.0 \mathrm{~kg}$. Ten polarization maintaining optical fibers (yellow) guide the laser beams to the cesium tube. All diode lasers are mounted on Peltier coolers for temperature regulation within $2 \mathrm{mK}$. (courtesy EADS SODERN). Right: The PHARAO engineering model cesium tube without the two external magnetic shields. The volume is $990 \times$ $336 \times 444 \mathrm{~mm}^{3}$ and the total mass is $44 \mathrm{~kg}$

optical local oscillator, PHARAO will provide a reduced frequency instability of $3 \times 10^{-14}(\tau / \mathrm{s})^{-1 / 2}$. This corresponds to $1 \times 10^{-15}$ at $1,000 \mathrm{~s}$ and $3 \times 10^{-16}$ at $10^{4} \mathrm{~s}$.

\subsubsection{Ion optical clock}

The ${ }^{171} \mathrm{Yb}^{+}$ion clock, shown in Figs. 6 and 7, provides an atomic frequency reference based on a single ${ }^{171} \mathrm{Yb}^{+}$ion confined within an RF electric end-cap trap (similar to a Paul trap) within an ultra high vacuum (UHV) chamber, and laser cooled to $\sim 1 \mathrm{mK}$, close to the Doppler cooling limit. As a result, the ion's first order Doppler motion is completely removed, and the ion experiences little collisional perturbation from its environment. Under these conditions, the narrow linewidth ${ }^{2} S_{1 / 2}\left(F=0, m_{F}=0\right)-{ }^{2} D_{3 / 2}\left(F=2, m_{F}=0\right)$ quadrupole


Fig. 6 Left: Simplified level scheme of ${ }^{171} \mathrm{Yb}^{+}$showing relevant cooling (blue), auxiliary (red), quadrupole clock (green) and octupole clock (dark blue) transitions. $\delta v_{\text {nat }}$ is the natural linewidth of the clock transition. Middle: close-up of Paul-type RF trap (PTB Braunschweig); the ion is trapped in the hole (diameter $1.4 \mathrm{~mm}$ ). Right: Complete ion trap with vacuum flange; height $10 \mathrm{~cm}$ (NPL Teddington) 




$876 \mathrm{~nm}$ clock laser wave from MOLO

Fig. 7 Simplified schematic of the single-ion optical clock unit. $A O M$ : acousto-optic frequency shifter. $P M T$ : photomultiplier tube; $E C$ : endcap; $T$ : temperature stabilization system, $B$ : magnetic field

clock transition at $435.5 \mathrm{~nm}$ can be interrogated by spectrally narrow and stable clock laser light. A diagram of the simplified energy level scheme for ${ }^{171} \mathrm{Yb}^{+}$ showing the relevant cooling, auxiliary and clock transitions is given in Fig. 6. All these wavelengths are provided by diode lasers, operated in fundamental or frequency doubled mode. The clock laser light is obtained from the $871 \mathrm{~nm}$ wave provided by the MOLO unit (see Section 4.2.3) after frequency-doubling in single pass by a $\mathrm{KNbO}_{3}$ nonlinear-optical crystal to $435.5 \mathrm{~nm}$. The clock transition spectral profile is observed by stepping the clock laser frequency by means of an acousto-optic frequency shifter (AOM in Fig. 7), and recording the statistics of the quantum jumps in cooling laser induced fluorescence as a function of this frequency. Under clock operation, the AOM repeatedly steps back and forward between the half-intensity points on the transition profile, monitoring the signal imbalance between these points. Any detected imbalance is servo-corrected to zero by feedback to the AOM.

In detail, the optical clock architecture will comprise (Fig. 7):

- an RF end-cap trap for ionising and confining a single ${ }^{171} \mathrm{Yb}^{+}$ion within an ultra-high vacuum chamber pumped by a small ion pump and nonevaporable getter pump. The trap is driven by means of an ac voltage of a few hundred volts at a drive frequency of $\sim 10 \mathrm{MHz}$. A small oven with several $\mathrm{mg}$ of ${ }^{171} \mathrm{Yb}$ isotope is heated to provide a low flux of $\mathrm{Yb}$ atoms, from which single ions can be ionised by electron bombardment from a hot wire filament within the trap potential well. Low voltage dc is applied 
to additional electrodes to position the ion precisely at trap potential centre in order to minimise the ion's micromotion at the drive frequency. This needs automatic periodic monitoring and correction, especially in the period after re-loading an ion into the trap. External magnetic field coils in three orthogonal axes allow the nulling of external fields, and setting of a fixed field of $\sim 1 \mu \mathrm{T}$. The trap and coils are surrounded by mu-metal shielding to minimise external field changes during the orbit. A level of temperature control of $1 \mathrm{~K}$ of the region surrounding the trap is required for maintaining the blackbody-induced frequency shift uncertainty with temperature at the $10^{-17}$ level;

- a laser platform to provide frequency doubling of an extended cavity $739 \mathrm{~nm}$ diode laser for Doppler cooling of the ion on the ${ }^{2} S_{1 / 2}(F=1)-$ ${ }^{2} P_{1 / 2}(F=0)$ dipole transition at $369.5 \mathrm{~nm}$. Light from an amplified diode laser device, frequency-doubled in a periodically poled $\mathrm{LiTaO}_{3}$ crystal in single-pass will be used. Typical laser powers used for driving the cooling transition below saturation are about $2 \mu \mathrm{W}$ for a beam waist in the trap of about $50 \mu \mathrm{m}$. By modulation of the injection current of the $739 \mathrm{~nm}$ diode laser at $14.7 \mathrm{GHz}$, a sideband is generated that excites the ${ }^{2} S_{1 / 2}(F=$ $0) \rightarrow^{2} P_{1 / 2}(F=1)$ "repumper" transition in order to avoid optical pumping between the ground hyperfine states;

- The platform also houses the auxiliary lasers at 935 and $638 \mathrm{~nm}$. The $935 \mathrm{~nm}$ diode provides repumping of the ion from the ${ }^{2} D_{3 / 2}(F=1)$ metastable level after occasional branching decays to this level during the cooling sequence. The $638 \mathrm{~nm}$ diode allows fast recovery of the ion from the very-long-lived ${ }^{2} \mathrm{~F}_{7 / 2}$ metastable state after very occasional collisional decay to that state. Currently, extended cavity lasers are forseen, but distributed-feedback lasers may be available in the near future;

- a high NA lens imaging system and photomultiplier detection system to record the statistics of $369 \mathrm{~nm}$ fluorescence quantum jumps as a function of clock laser frequency step;

- a fibre system to deliver the various cooling, auxiliary and clock light from source to trap, making use of achromatic doublets where necessary at the fibre-free space interface for launching into the trap;

- a monitoring and control processor, which provides the primary cooling and clock laser pulse, magnetic field and detection sequencing to observe and lock to the ion clock transition frequency. The processor also monitors frequency and amplitude data necessary to determine normal laser and ion operational conditions and initiate resetting and recovery algorithms where necessary, and laser unit failure. The actual magnetic field present on the atomic volume is determined and actively stabilized, so that an inaccuracy at the level of $1 \times 10^{-17}$ can be achieved;

- a redundancy level of at least two units for cooling, clock and repumper lasers, plus similar for frequency doubling crystals. All redundancy units for each wavelength will be fibre multiplexed as standard, allowing redundant unit activation on determination of prior unit failure; 
For an optimized, quantum-projection-noise-limited, performance of the single-ion clock, Ramsey interrogation with a cycle time equal to the lifetime of the metastable $\mathrm{D}_{3 / 2}$ level of $50 \mathrm{~ms}$ will be performed, leading to an Allan deviation of about $2.7 \times 10^{-15}(\tau / \mathrm{s})^{-1 / 2}$. This is $\sim 4.5 \times 10^{-17}$ at $1 \mathrm{~h}$, satisfying required stabilities for the science objectives. Current laboratory results are a stability of $1 \times 10^{-15}$ at $30 \mathrm{~s}$, averaging down to $3 \times 10^{-16}$ at $1,000 \mathrm{~s}$ (PTB, Germany). Also, comparisons between two independent traps showed agreement at the level of several parts in $10^{-16}$ over a series of eight measurement runs.

The ${ }^{171} \mathrm{Yb}^{+}$ion clock offers a second option, namely the ${ }^{2} S_{1 / 2}{ }^{2} F_{7 / 2}$ octupole transition at $476 \mathrm{~nm}$ (see Table 1 and Fig. 6) [48]. It has an extremely long upper state lifetime, low electric quadrupole and second order Zeeman systematic shifts and therefore is a candidate for an even higher performance clock, if a clock laser system with lower instability than the above is provided. Apart from the clock laser, it shares the laser system of the quadrupole clock. Thus, with moderate additional resources, the $\mathrm{Yb}^{+}$ion apparatus could provide two optical clocks in a single package, and specifically perform direct frequency comparisons between the quadrupole and the octupole transition, with a large sensitivity $A_{\alpha}$.

The ion clock will operate under microprocessor control providing the control algorithms for the integrated pulse sequencing for cooling, repumping, clock interrogation, magnetic field switching, cooling fluorescence monitoring. The controller will also provide error flags for critical processes and system reset and correction where necessary. This will include ion re-loading and micromotion reduction algorithms. Automatic monitoring of laser power and spectral quality, with signal re-optimisation or laser failure determination is required. Redundant laser units will be pre-aligned and multiplexed into the fibre delivery to the trap so that redundant units can be readily activated in the case of diode laser failure.

\subsubsection{Microwave-optical local oscillator (MOLO)}

This oscillator provides two ultrastable frequency outputs, one in the optical region, the other in the microwave region, the two frequencies being coherently related. The MOLO is composed of three subunits: the clock laser, the reference cavity, and the frequency comb, see Fig. 8.

The clock laser subunit delivers the light for the excitation of the optical clock transition. It is generated by an extended-cavity diode laser at a wavelength of $871 \mathrm{~nm}$. Most of the output wave of this laser is sent to the ion clock. The laser subunit, containing two lasers for redundancy, will require $3 \mathrm{~kg}, 31,15 \mathrm{~W}$. The frequency instability of the clock laser must be superior to the minimum clock instability $2.7 \times 10^{-15}(\tau / \mathrm{s})^{-1 / 2}$ for $\tau$ up to the time constant ( $\sim 10 \mathrm{~s}$ ) of the servo system that locks the laser to the ion's resonance signal. In order to achieve this, the laser frequency is stabilized to the reference 


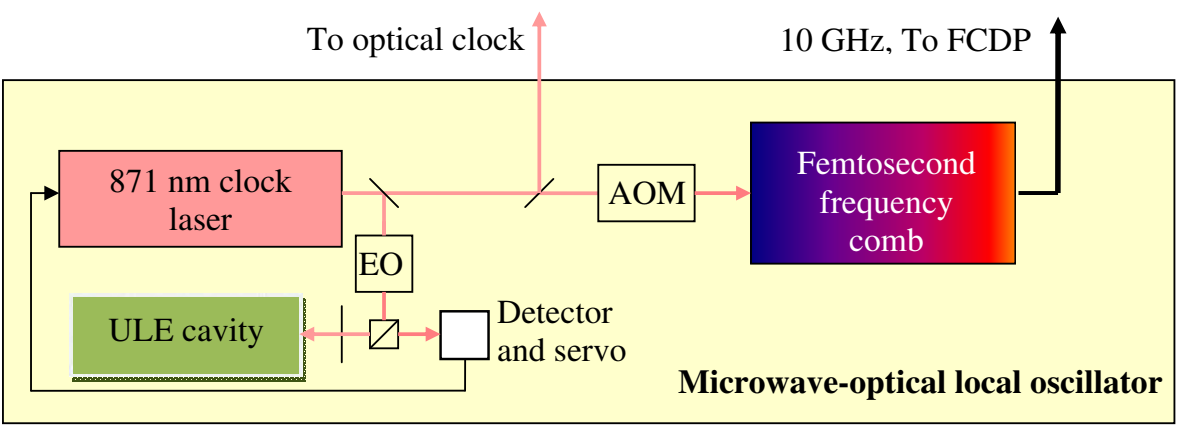

Fig. 8 Microwave-optical local oscillator (MOLO), consisting of clock laser, reference ULE cavity and femtosecond frequency comb (OFC). Radiofrequency inputs and outputs are not shown. EO: electro-optic modulator; $U L E$ : ultra-low expansion; $A O M$ : frequency shifter

cavity, where the Pound-Drever-Hall method is used. About $10 \mu \mathrm{W}$ of the laser output are used for this.

The system will exhibit $\sim 0.4 \mathrm{~Hz}\left(\sim 1 \times 10^{-15}\right.$ relative $)$ linewidth and a frequency drift $<0.1 \mathrm{~Hz} / \mathrm{s}$. The cavity consists of two high-reflectivity mirrors optically contacted to a cylinder of diameter $70 \mathrm{~mm}$, length $150 \mathrm{~mm}$, made of ULE (a glass exhibiting ultra low thermal expansion coefficient) with a cavity finesse of about 300,000 . Residual vibrations of the satellite $\left(a_{\mathrm{rms}}<1 \times 10^{-6} \mathrm{~g}\right)$ should not be a limiting factor to the desired linewidth; for safety margin, an optimized cavity support can reduce the sensitivity to accelerations. For acoustic and thermal isolation the cavity resides inside an aluminium vacuum chamber equipped with a small $(3 \mathrm{l} / \mathrm{s})$ ion-getter pump. Two stages of polished aluminium shields are implemented around the cavity, which are actively temperature stabilized, so that the cavity is precisely kept at the zero crossing temperature of the ULE thermal expansion coefficient. Heat application and removal is by thermoelectric elements between the shield and the vacuum chamber. Additionally, the entire vacuum chamber is temperature-stabilized.

During transportation and launch, the cavity will be rigidly fixed inside the vacuum chamber by pressing on it from the sides by piezo-mechanical actuators, allowing accelerations of several g. After bringing the satellite on the orbit, the actuators will be released.

The optical setup for frequency stabilization will be a classic Pound-DreverHall scheme in reflection, using an electro-optic modulator (EO). Coupling of the clock laser radiation to the cavity is via single-mode polarization maintaining optical fiber. The fiber in- and out-couplers are mounted on miniature piezo-motor driven multiaxis translation stages. Microprocessor control of the translation stages performs a laser-to-fiber incoupling stabilization and laser-to-cavity mode-matching optimization (positioning inaccuracy: $<10 \mu \mathrm{m}$, alignment inaccuracy: $<10 \mu \mathrm{rad})$.

The frequency stability of the clock laser will be limited by thermal $1 / \mathrm{f}$ noise in the mirrors, with an fractional Allan deviation slightly below $1 \times 10^{-15}$ from $1 \mathrm{~s}$ to a few $10 \mathrm{~s}$. Resources, including control electronics, are $151,12 \mathrm{~kg}, 8 \mathrm{~W}$. 
A femtosecond fiber laser based optical frequency comb generator (OFC, Fig. 8) will be used for the clock frequency comparisons and to obtain a spectrally ultrapure microwave local oscillator output for the operation of PHARAO, for the microwave link and for the GNSS receiver. The femtosecond laser is based on erbium-doped fiber, pumped by fiber-coupled laser diodes. It emits $100 \mathrm{fs}$ pulses at a repetition rate of $250 \mathrm{MHz}$, with an average power of $100 \mathrm{~mW}$. The emission spectrum is centered around 1,560 nm. Spectral broadening to more than one octave is obtained via nonlinear interaction in a microstructure fibre, so that the range from about 1,050 to $2,100 \mathrm{~nm}$ is covered.

Second harmonic generation of a portion of the broadened spectrum allows obtaining the required light for the measurement of the clock laser frequency at $871 \mathrm{~nm}$. The OFC will be referenced to the clock laser frequency (after introduction a controllable shift via the frequency shifter AOM), by making the carrier envelope offset frequency vanish, so that the repetition rate $f_{r}$ is a high-order subharmonic of the optical clock reference frequency $f_{1}=m_{1} f_{r}$, The link between the optical and the microwave domain is then simply the distribution of the repetition rate frequency, or, more conveniently, a high (order 40) harmonic of the comb repetition rate, $10 \mathrm{GHz}$.

It has been demonstrated by several groups that frequency comparison and frequency division with an OFC can be performed to very high accuracy and stability, beyond the limitations imposed by the optical clocks themselves. For example, a fiber laser OFC with an instability (Allan deviation) of $6 \times 10^{-17}$ in $1 \mathrm{~s}$ has been demonstrated [51]. OFCs based on fiber technology possess good long-term reliability and high power efficiency. The projection for a space qualified OFC, based on current industrial terrestrial technology, is $16 \mathrm{~kg}$ mass, 201 volume, and $38 \mathrm{~W}$ power requirement.

\subsubsection{Frequency comparison and distribution package (FCDP)}

This package distributes the ultra-stable $10 \mathrm{GHz}$ reference frequency output of the MOLO to the MWL, performs frequency comparisons between MOLO and PHARAO, and generates a variety of different reference signals for the individual instruments, including the $250 \mathrm{MHz}$ reference clock to the OFC. All signals are coherent to the MOLO. In the FCDP, a USO is locked to the MOLO signal by a digital PLL. A divider chain generates timing pulses (1 pps, 1,000 pps etc) and keeps a continuous local time scale. From the digital phase comparison data, the phase noise density spectrum between the USO and the MOLO is available by telemetry (TM) to assess the overall frequency purity in-flight.

As the FCDP distributes the full performance reference from the MOLO directly to the MWL, there is minimum impact of the FCDP on overall clock comparison performance. The USO-phase lock loop uses a similar design as for ACES-FCDP. The digital PLL with linear phase detector allows the USO to be slightly offset from its reference, which is selectable by ground command. This results in an accurate on-board time scale despite any offset 
of the MOLO reference frequency, which can be synchronised to UTC. The frequency synthesis chain re-uses modules developed for ACES-FCDP and their frequency inaccuracy. Expected relative uncertainty (Allan deviation) of the frequency comparison between two signals provided to the FCDP is $4.7 \times 10^{-17}$ at $1,000 \mathrm{~s}, 9.5 \times 10^{-18}$ at $10,000 \mathrm{~s}$.

During non-availability of the MOLO, i.e. during system start-up and contingency conditions, the USO runs as flywheel (i.e. it is unlocked, with instability $<1.5 \times 10^{-13}$ from 1 to $100 \mathrm{~s}$ ). It will still provide useable, but less accurate references to the instruments, including the reference to OFC. Common view ground-to-ground time- and frequency comparison and supply of the remaining instruments remain possible, at the USO provided accuracy.

Mass, volume and power consumption are $8 \mathrm{~kg}, 71,12 \mathrm{~W}$. Control/data rate is $10 \mathrm{kB} /$ day, $50 \mathrm{kB} /$ day (FCDP alone).

\subsubsection{Microwave link (MWL)}

The microwave link is a two-way triple-frequency microwave link (Fig. 9). It is designed to perform accurate frequency comparison as well as time-transfer

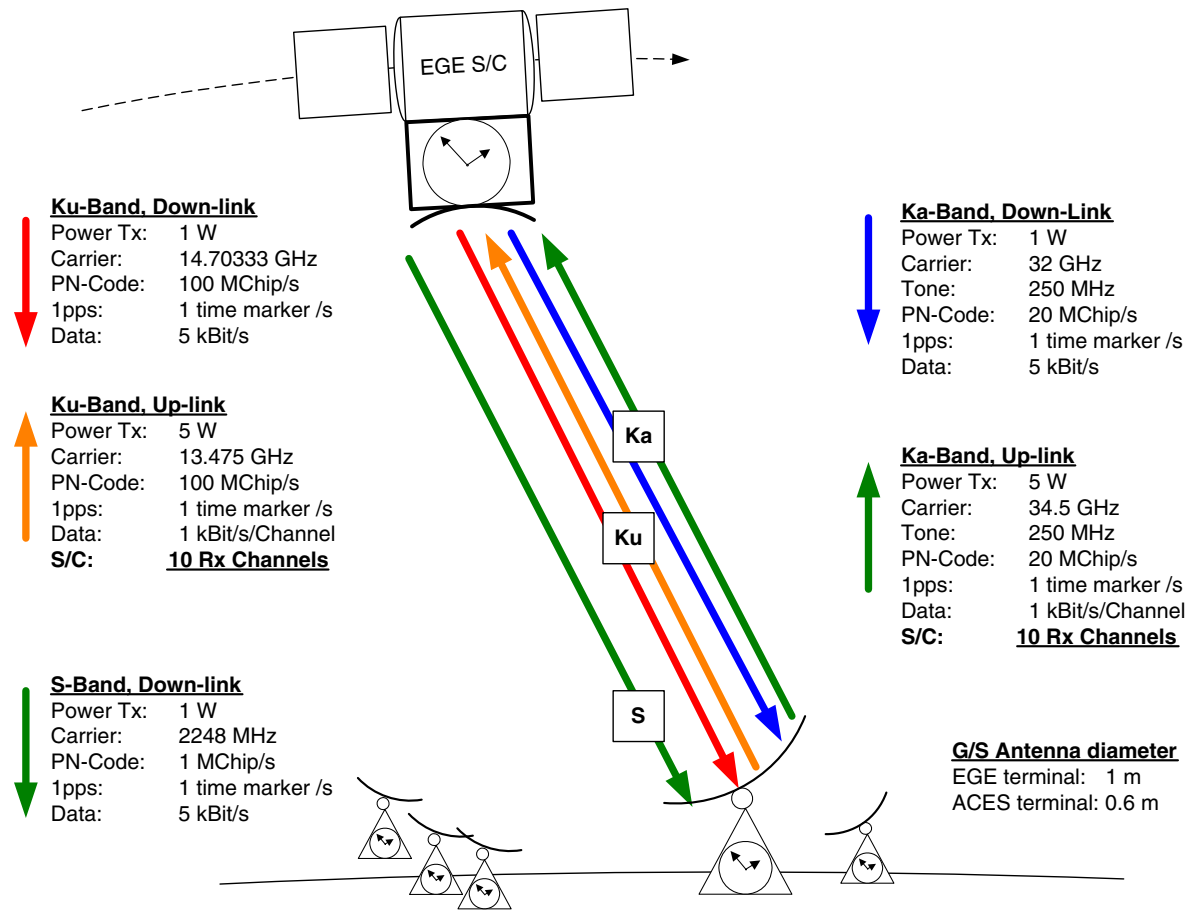

Fig. 9 EGE microwave two-way link concept and characteristics. See text for a description. The link will be able to perform frequency comparisons with up to ten ground stations simultaneously (only one active comparison is shown). pps: pulse per second; $T x$ : transmitter. $S / C$ : spacecraft 
between on-board and on-ground clocks. Two-way operation allows for compensation of the effects due to spacecraft location and its motion relative to ground. Its two bi-directional links operate in Ka-band $(32 / 34.5 \mathrm{GHz})$ and Ku-band (14.7/13.5 GHz). The high carrier frequency of the up- and downlinks allows for a noticeable reduction of the ionospheric delay. A third downlink channel is in S-band $(2.2 \mathrm{GHz})$ and is used to determine the ionosphere total electron content. MWL uses a combination of carrier phase and code phase data to measure with high precision the absolute ionospheric delay as well as ionospheric delay variations during a ground contact. The use of three frequency bands allows to resolve third order ionospheric terms. Key modulation parameters are: Ka band: Tone $250 \mathrm{MHz}$; Ku band: PN $100 \mathrm{MChip} / \mathrm{s}$; S-band: 1 MChip/s.

The EGE-MWL concept is a considerably improved version of the ACESMWL. Microwave carrier generation is directly from the $10 \mathrm{GHz}$ microwave output available from the MOLO and having exceptionally low close-in phase noise, without intermediate frequency dividers or other complex synthesiser stages commonly used in RF transponders. The high frequency carriers are key to achieve the desired frequency comparison accuracy.

The Ka-band carrier is generated by tripling the FCDP-generated signal. The Ku-band is subsequently generated by division by two using a very stable regenerative divider. Finally, the assigned radio frequency is obtained by adding a low frequency signal using an image reject mixer. A mixed analog-digital receiver design will enhance its performance by use of a flexible digital signal processing and digital control loops. The number of simultaneous ground contacts is increased from four (as in the ACES mission) to ten or more using the digital concept, while the number of physical receiver channels per band is reduced from four to two, one for operation to ground and one for inflight calibration. The concept allows adaptation of receiver loop parameters to optimise for the different portions of a highly elliptic orbit while reducing systematic uncertainties due to high Doppler and Doppler rate. Real-time data exchange to ground terminals is by additional data modulation on all RF signals. The spacecraft carries two different small antennae for perigee and for apogee, with gains of $8 \mathrm{dBi}, 20 \mathrm{dBi}$, beamwidths $100^{\circ}$ (full earth coverage), $14^{\circ}$, respectively; power is $1 \mathrm{~W}$ for each.

The MWL end-to-end performance between the attached clocks, including space- and ground terminals is predicted to be a relative Allan deviation of $1 \times 10^{-16}$ at $1,000 \mathrm{~s}$ (perigee) and $1 \times 10^{-17}$ at $10,000 \mathrm{~s}$ (apogee). These values are based on conservative implementation losses and other instrumental losses, assumed as $6 \mathrm{~dB}$ at apogee and $10 \mathrm{~dB}$ at perigee, including additional losses due to atmosphere. An attitude knowledge of $1^{\circ}$ is required.

The MWL link will also support precise orbitography-complementary to the GNSS receiver. Generation of ranging data is simultaneous to two-way time transfer and is available under all weather conditions. Orbit determination accuracy is expected to be better than $1 \mathrm{~cm}$, well matching EGE clock requirements. The value is expected based on demonstrated results from other missions (METEOR, ERS-2, TOPEX/POSEIDON). Laser ranging to 
the CCR array is used a few times during the mission to calibrate the MWL ranging.

In case of non-availability of the ultrapure signal from MOLO, an additional $10 \mathrm{GHz}$ output is available from the FCDP to support ground-to-ground clock comparison in common-view mode, even after the nominal mission period, when the neither the clocks nor MOLO may be available any longer. Based on the PRARE experiment's 12 years of operation, this can be assumed for the FCDP/MWL combination as well. The potential extended operational period makes the proposed configuration particularly attractive to metrological laboratories, that rely on availability of long-lasting data in support of the generation of UTC.

The resources of MWL are as follows: mass: $18 \mathrm{~kg}$, volume: 18 1, power: $55 \mathrm{~W}, \mathrm{TC}: 2 \mathrm{kB} /$ day, TM: $120 \mathrm{MB} /$ day.

\subsubsection{Auxiliary units}

The star camera assembly on the EGE spacecraft will provide real-time attitude data primarily for the orientation of the spacecraft (with accuracy of $1^{\circ}$ in real-time) and the proper reduction of the GNSS/SLR data.

A GNSS receiver is used for the precise orbit determination, time and frequency transfer, coarse positioning $(<1 \mathrm{~m})$ for real time use by the Attitude and Orbit Control as well as the back-up for time tagging of all payload data. For the time and frequency transfer, the receiver is nominally using the onboard frequency reference. The receiver assembly includes three special antennas. By the year 2015 more than 100 GNSS satellites are expected to be available for precise orbit determination, permitting orbit inaccuracy of 1$2 \mathrm{~cm}$ RMS for altitudes up to approx. 10,000 km. At apogee, a combination of GNSS signals and MWL ranging will be used, with expected inaccuracy of $10 \mathrm{~cm}$.

\section{Basic spacecraft specifications}

The mission foresees a single spacecraft with a mission duration limited to approx. 3 years for cost reasons. Attitude control along three axes with less than $1^{\circ}$ error on a timescale of $1,000 \mathrm{~s}$ is required. The control can be performed with cold gas thrusters, magnetotorquers, or reaction wheels. The selection will be done on the basis of the vibrations induced by these systems (desired level: $a_{\mathrm{rms}}<10^{-6} \mathrm{~g}$ ).

Cold gas thrusters or monopropellant thrusters will be required to maintain the orbit parameters during the mission by small orbit correction manoeuvres and will also guarantee a proper tuning of the orbit parameters after the release from the launcher. The ground segment (telemetry, control, and data transfer) could be via non-ESA stations, user-operated, to save costs.

Further resources are indicated in Table 2. 
The satellite bus design drivers are: nadir-pointing microwave antennas, thermal stability for the payload instruments, no strong magnetic sources, low microvibrations, harsh radiation environment due to crossing of the radiation belts once per orbit, power demand by science payload (with the possibility to reduce power demand by stand-by of some components during specific mission phases, e.g. eclipse), mass limit due to required high altitude with low cost launch.

The satellite bus design can be based on established platforms such as FLEXBUS or PRIMA, used on several past missions. Their design philosophy differs, one having body-mounted solar arrays, the other wings. The latter can provide larger amounts of power. The vibration level induced by the panels when they are fixed and the duration of solar array drive periods per orbit will have to be considered in the selection. The FLEXBUS concept was used for a mission already requiring a low level of residual accelerations, and the platform is optimised for this parameter.

For most of the satellite components one can consider using components and electronic units from navigation or communication satellites which typically cruise at altitudes between 15,000 and $25,000 \mathrm{~km}$ altitude. LISA Pathfinder, which will cross the radiation belts during spiralling to the Lagrange point, will face similar problems concerning radiation as EGE as well as XMM, which is in a Molniya-type orbit with similar radiation characteristics.

The instruments will therefore be located in the satellite structure such as to realise ionizing radiation shielding by the spacecraft structure and nonsensitive equipment to the maximum extent possible; the need for additional radiation shielding (including trapped proton radiation) for especially sensitive parts of the instruments must be determined by ground tests. It should be mentioned that an orbit with higher perigee and less radiation dose is also an option, as it leads to a tolerable reduction of the science goals.

The required $\pm 3^{\circ} \mathrm{C}$ temperature stability at the interface to the payload enclosure is feasible. The thermal control will employ the usual means (an enclosure with high thermal inertia, heaters and a suitable radiator). The location of the radiator will need a special study given the non-standard orbit.

Magnetic field present during the mission can affect the properties of the onboard clock. Using SPENVIS, the magnetic field has been calculated along the spacecraft orbit: the field intensity presents maxima around $0.2 \mathrm{G}$ and a minimum value of the order of $10^{-3} \mathrm{G}$. As discussed above, this is not a limitation to the instrument performance.

\section{Discussion and conclusion}

The accuracy of the measurements depends crucially on the stability and accuracy of the clocks. While the performance of ground clocks (even those eventually deployed in new ground stations dedicated to EGE) will likely improve significantly from the present level, the space clock design will be "frozen" approx. 10 years before data acquisition. Thus, their performance 
level will be significantly below the future ground station clock performance. As specification, we have chosen a performance level that has been demonstrated with laboratory breadboards recently. We see no fundamental reason that this laboratory performance cannot also be achieved in a space ion optical clock. The experience with the PHARAO cold atom clock has shown that an engineering model of a laser-based cold atom clock has a performance similar to that of a laboratory prototype. The perturbation of the ion clock frequency due to magnetic fields along the orbit can be modelled and suppressed with shielding and the residual effects measured and compensated for. The moderate temperature stability $\left( \pm 3^{\circ} \mathrm{C}\right)$ to be provided in the experimental bay can be achieved with thermal shields. Accurate temperature control of particular units is done by active stabilization of the units themselves.

One important aspect is radiation, which may affect all optical and trap components as well as the lasers and reference cavity. The radiation environment on the EGE orbit is significantly harsher than the near-earth ISS orbit, on which ACES will operate. The radiation effects must therefore be characterized comprehensively with appropriate tests and then the radiation shield for the EGE instruments designed.

In summary, the Einstein Gravity Explorer mission (EGE) is a satellite mission aimed at a precise measurement of the properties of space-time using atomic clocks. By testing one of the most fundamental predictions of Einstein's Theory of General Relativity, the gravitational redshift, and thereby searching for quantum effects in gravity, it explores one of the most important frontiers in fundamental physics. The EGE mission reaches beyond fundamental physics, providing an important spin-off in geophysics. Furthermore, the technological aspects of the mission are ground-breaking for future time-keeping and reference frame systems in space, and thus are also expected to be of wide use to science and technology in general. The payload of the mission has been conceived as a compromise between cost and performance, needing to fit the cost envelope of an ESA medium-class mission. ${ }^{1}$

Acknowledgement We thank S. Cesare for helpful information.

\section{References}

1. Will, C.M.: The confrontation between general relativity and experiment. Living Rev. Relativ. 9, 3 (2006)

2. Ni, W.-T.: Empirical foundations of the relativistic gravity. Int. J. Mod. Phys. D 14, 901 (2005). doi:10.1142/S0218271805007139

\footnotetext{
${ }^{1}$ Finally, we point out that a spacecraft with a significantly reduced and thus lower-cost payload (no clocks; only MOLO, FCDP, MWL) on a circular orbit of appropriate height could be used for a long-duration earth science mission dedicated specifically to the measurement of the geopotential.
} 
3. Bertotti, B., et al.: A test of general relativity using radio links with the Cassini spacecraft. Nature 425, 374 (2003). doi:10.1038/nature01997

4. Vessot, R.C.F., et al.: Test of relativistic gravitation with a space-borne hydrogen maser. Phys. Rev. Lett. 45, 2801 (1980). doi:10.1103/PhysRevLett.45.2081

5. Laurent, P., et al.: Design of the cold atom PHARAO space clock and initial test results. Appl. Phys. B 84, 683 (2006). doi:10.1007/s00340-006-2396-6

6. Cacciapuoti, L., et al.: Atomic clock ensemble in space: scientific objectives and mission status. Nucl. Phys. B Proc. Suppl. 166, 303 (2007). doi:10.1016/j.nuclphysbps.2006.12.033

7. Flambaum, V.V., Kozlov, M.G.: Limit on the cosmological variation of $m_{p} / m_{e}$ from the Inversion Spectrum of Ammonia. Phys. Rev. Lett. 98, 240801 (2007). doi: 10.1103/PhysRevLett.98.240801

8. Reynold, E., et al.: Indication of a cosmological variation of the proton-electron mass ratio based on laboratory measurement and reanalysis of $\mathrm{H}_{2}$ spectra. Phys. Rev. Lett. 96, 151101 (2006). doi:10.1103/PhysRevLett.96.151101

9. Tzanavaris, T., et al.: Limits on variations in fundamental constants from $21-\mathrm{cm}$ and ultraviolet quasar absorption lines. Phys. Rev. Lett. 95, 041301 (2005). doi: 10.1103/ PhysRevLett.95.041301

10. Kanekar, N., et al.: Constraints on changes in fundamental constants from a cosmologically distant $\mathrm{OH}$ absorber or emitter. Phys. Rev. Lett. 95, 261301 (2005). doi: 10.1103/PhysRevLett.95.261301

11. Flambaum, V.V.: Variation of the fundamental constants: theory and observations. Int. J. Mod. Phys. A 22, 4937 (2007). doi:10.1142/S0217751X07038293

12. Dent, T., et al.: Primordial nucleosynthesis as a probe of fundamental physics parameters. Phys. Rev. D Part. Fields Gravit. Cosmol. 76, 063513 (2007). doi:10.1103/PhysRevD.76.063513

13. Dmitriev, V.F., et al.: Cosmological variation of the deuteron binding energy, strong interaction, and quark masses from big bang uncleosynthesis. Phys. Rev. D 69, 063506 (2004) and refs. therein

14. Wetterich, C.: Crossover quintessence and cosmological history of fundamental "constants". Phys. Lett. B 561, 10 (2003). doi:10.1016/S0370-2693(03)00383-6

15. Wetterich, C.: Probing quintessence with time variation of couplings. Astropart. Phys. 10, 2 (2003)

16. Sandvik, H.B., et al.: A simple cosmology with a varying fine structure constant. Phys. Rev. Lett. 88, 031302 (2002). doi:10.1103/PhysRevLett.88.031302

17. Fortier, T.M., et al.: Precision atomic spectroscopy for improved limits on variation of the fine structure constant and local position invariance. PRL 98, 070801 (2007). doi:10.1103/PhysRevLett.98.070801

18. Ashby, N., et al.: Testing local position invariance with four cesium-fountain primary frequency standards and four NIST hydrogen masers. PRL 98, 070802 (2007). doi: 10.1103/PhysRevLett.98.070802

19. Hoffmann, B.: Noon-midnight red shift. Phys. Rev. 121, 337 (1961). doi:10.1103/ PhysRev.121.337

20. LoPresto, J.C. et al.: Solar gravitational redshift from the infrared oxygen triplet. Astrophys. J. 376, 757 (1991)

21. Krisher, T.P., et al.: The Galileo solar redshift experiment. Phys. Rev. Lett. 70, 2213 (1993). doi:10.1103/PhysRevLett.70.2213

22. Soffel, M., et al.: The IAU 2000 resolutions for astrometry, celestial mechanics, and metrology in the relativistic framework: explanatory supplement. Astron. J. 126, 2687 (2003). doi: $10.1086 / 378162$

23. Moyer, T.D.: Formulation for Observed and Computed Values of Deep Space Network Data Types for Navigation. Wiley, New York (2003)

24. IERS Conventions: In: McCarthy, D.D., Petit, G. (eds.) IERS Technical Note No. 32. Frankfurt am Main: Verlag des Bundesamts für Kartographie und Geodäsie, 2004 (2003)

25. Kaplan, G.H.: The IAU Resolutions on Astronomical Reference Systems, Time Scales, and Earth Rotation Models, U.S. Naval Circular No. 179 (Washington: USNO), 2005. astro$\mathrm{ph} / 0602086$ (2004)

26. Blanchet, L., et al.: Relativistic theory for time and frequency transfer to order $\mathrm{c}^{-3}$. Astron. Astrophys. 370, 320 (2000). doi:10.1051/0004-6361:20010233 
27. Linet, B., Teyssandier, P.: Time transfer and frequency shift to the order $1 / \mathrm{c}^{4}$ in the field of an axisymmetric rotating body. Phys. Rev. D Part. Fields 66, 024045 (2002). doi:10.1103/ PhysRevD.66.024045

28. Le Poncin-Lafitte, C., Lambert, S.B.: Testing general relativity with the ACES mission. arxiv:astro-ph/0610463v1

29. Le Poncin-Lafitte, C., Lambert, S.B.: Numerical study of relativistic frequency shift for the cold-atom clock experiment in space. Class. Quantum Gravity 24, 801 (2007)

30. Reinhardt, S., et al.: Test of relativistic time dilation with fast optical atomic clocks at different velocities. Nat. Phys. 3, 861 (2007). doi:10.1038/nphys 778

31. Ehlers, G., Lämmerzahl, C. (eds.): Special Relativity: Will It Survive the Next 101 Years? Springer (2006)

32. Kostelecky, V.A. (ed.) Proc. First, Second, and Third Meetings on CPT and Lorentz Symmetry. (World Scientific, 1999, 2002, 2005)

33. Kostelecky, A., Lane, C.D.: Constraints on Lorentz violation from clock comparison experiments. Phys. Rev. D Part. Fields 60, 116010 (1999). doi:10.1103/ PhysRevD.60.116010

34. Mansouri, R., Sexl, R.U.: A test theory of special relativity: III. Second-order tests. Gen. Relativ. Gravit. 8, 809 (1977). doi:10.1007/BF00759585

35. Braxmaier, C., et al.: Tests of relativity using a cryogenic optical resonator. Phys. Rev. Lett. 88, 010401 (2002). doi:10.1103/PhysRevLett.88.010401

36. Lämmerzahl, C., et al.: OPTIS: a satellite-based test of special and general relativity. Class. Quantum Gravity 18, 2499 (2001). doi:10.1088/0264-9381/18/13/312

37. Scheithauer, S., Lämmerzahl, C.: Analytical solution for the deformation of a cylinder under tidal gravitational forces. Class. Quantum Gravity 23, 7273 (2006). doi:10.1088/ 0264-9381/23/24/006

38. Wolf, P., et al.: Cold atom clock test of Lorentz invariance in the matter sector. Phys. Rev. Lett. 96, 060801 (2006). doi:10.1103/PhysRevLett.96.060801

39. Fu, L.L., Cazenave, A.: Satellite Altimetry and Earth Sciences. Academic, New York (2001)

40. Reigber, C., et al.: An Earth gravity field model complete to degree and order 150 from Grace: Eigen-Grace02S. J. Geodynamics 39, 1 (2005)

41. Tapley, B.D., et al.: GRACE measurements of mass variability in the earth system. Science 305, 503 (2004). doi:10.1126/science.1099192

42. Rummel, R., et al.: IAG-Symposia, vol. 120, p. 261. Springer, Heidelberg (2000)

43. Bjerhammar, A.: On a relativistic geodesy. Bull. Geod. 59, 207 (1985). doi:10.1007/BF02520327

44. Francis C.R., et al.: The ERS-2 Spacecraft and its payload. ESA Bulletin-European Space Agency 83, 13 (1995). http://earth.esa.int/object/index.cfm?fobjectid=4007

45. Oskay, W.H., et al.: Single-atom optical clock with high accuracy. Phys. Rev. Lett. 97, 020801 (2006). doi:10.1103/PhysRevLett.97.020801

46. Schneider, T., et al.: Sub-Hertz optical frequency comparisons between two trapped ${ }^{171} \mathrm{Yb}^{+}$ ions. Phys. Rev. Lett. 94, 230801 (2005). doi:10.1103/PhysRevLett.94.230801

47. Margolis, H.S., et al:: Hertz-level measurement of the optical clock frequency in a single ${ }^{88} \mathrm{Sr}^{+}$ ion. Science 306, 1355 (2004). doi:10.1126/science.1105497

48. Tamm, C., et al.: ${ }^{171} \mathrm{Yb}^{+}$single-ion optical frequency standard at $688 \mathrm{THz}$. IEEE Trans. Instrum. Meas. 56, 601 (2007). doi:10.1109/TIM.2007.891140

49. Hosaka, K., et al.: An optical frequency standard based on the electric octupole transition in ${ }^{171} \mathrm{Yb}^{+}$. IEEE Trans. Instrum. Meas. 54, 759 (2005). doi:10.1109/TIM.2004.843319

50. Ludlow, A.D., et al.: Sr Lattice clock at $1 \times 10^{-16}$. Fractional uncertainty by remote optical evaluation with a Ca clock. Science 319, 1805 (2008). doi:10.1126/science.1153341

51. Hollberg, L.: From Quantum to Cosmos. Workshop Airlie (Va, USA), 6-10 July 2008

52. Newbury, N.R., et al.: Proc. 2007 Joint Mtg. IEEE Intl. Freq. Cont. Symp. and EFTF Conf. 980 (2007). http://tf.nist.gov/timefreq/general/pdf/2207.pdf 